\title{
Process Development and Large-Scale Synthesis of BTK Inhibitor BIIB068
}

\section{Supporting information}

\section{Table of Contents}

I. General Information

II. Analytical Spectra of Products

(1) ${ }^{1} \mathrm{HNMR},{ }^{13} \mathrm{CNMR}$, m.p and HRMS spectra of $\mathbf{1 0}$

(2) ${ }^{1} \mathrm{HNMR},{ }^{13} \mathrm{CNMR}$, m.p and HRMS spectra of BIIB068 (1)

(3) ${ }^{1} \mathrm{HNMR},{ }^{13} \mathrm{CNMR}, \mathrm{m} . \mathrm{p}$ and HRMS spectra of BIIB068 hemi-adipate salt (19)

(4) XRPD report of BIIB068 hemi-adipate salt (19)

III. Analytical Spectra of Impurities

(1) HPLC, ${ }^{1} \mathrm{HNMR}$ and HRMS spectra of 9

(2) HPLC, ${ }^{1}$ HNMR and HRMS spectra of $\mathbf{1 2}$

(3) LCMS spectra of impurity $\mathbf{1 8}$

(4) LCMS spectra of impurity $\mathbf{1 5}$

IV. Analytical Spectra of Reaction Optimization (7 to 10, table 1)

V. Single Crystal Structure of $\mathbf{1 9}$ 


\section{General Information}

All reagents and solvents were purchased from commercial suppliers and used without further purification. All reactions were carried out under nitrogen. ${ }^{1} \mathrm{H}$ NMR and ${ }^{13} \mathrm{C}$ NMR spectra were recorded on a Bruker Avance and Varian NMR Spectrometer. Chemical shifts were reported in ppm relative to the residual deuterated solvent for ${ }^{1} \mathrm{H}$ and the deuterated solvent for ${ }^{13} \mathrm{C}$. HPLC analysis was performed on Agilent 1290 instrument. LCMS analysis was performed on Agilent $1290 \mathrm{HPLC}+\mathrm{G} 6125$ MS instrument. XRPD was recorded on DX-2700BH instrument. m.p was recorded on METTLER MP80 $\left(1.0^{\circ} \mathrm{C} / \mathrm{min}\right)$ instrument with ChP2015 Vol. IV 0612 (Method I). HRMS analysis was performed on LC-QT instrument. All yields are uncorrected for purity.

\section{Analytical Spectra of Products}

(1) ${ }^{1} \mathrm{HNMR},{ }^{13} \mathrm{CNMR}$, m.p and HRMS spectra of $\mathbf{1 0}$

${ }^{1} \mathrm{HNMR}$ :

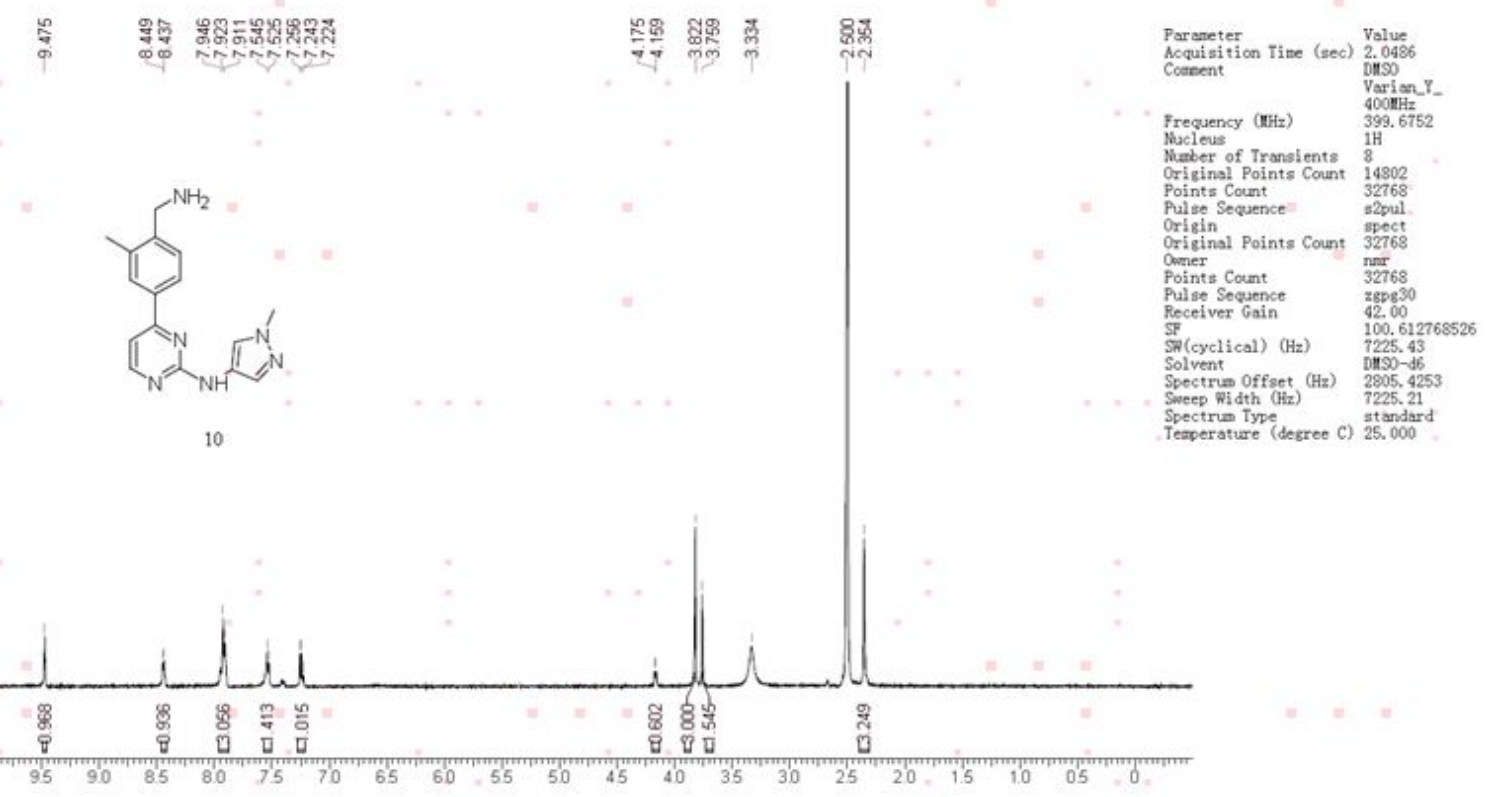


${ }^{13}$ CNMR:

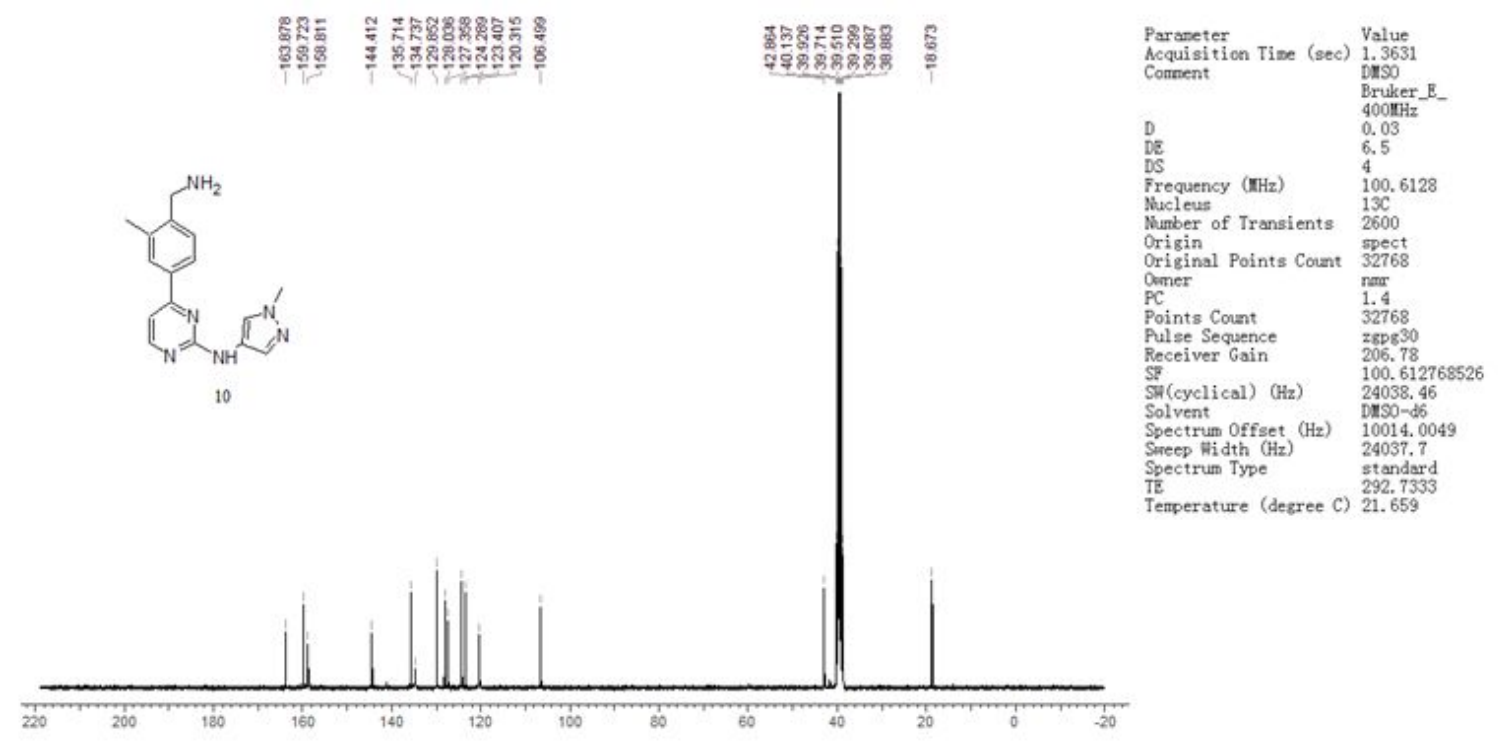

m.p:

Method : ChP2015 Vol.IV 0612 (Method I)

Instrument :METTLER MP80 $\left(1.0^{\circ} \mathrm{C} / \mathrm{min}\right)$

Results:

\begin{tabular}{|c|c|c|c|c|}
\hline Items & $1\left({ }^{\circ} \mathrm{C}\right)$ & $2\left({ }^{\circ} \mathrm{C}\right)$ & $3\left({ }^{\circ} \mathrm{C}\right)$ & $\bar{x}\left({ }^{\circ} \mathrm{C}\right)$ \\
\hline Melting Point & 146.1 & 146.0 & 145.7 & 145.9 \\
\hline
\end{tabular}

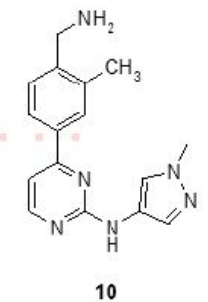

HRMS: 

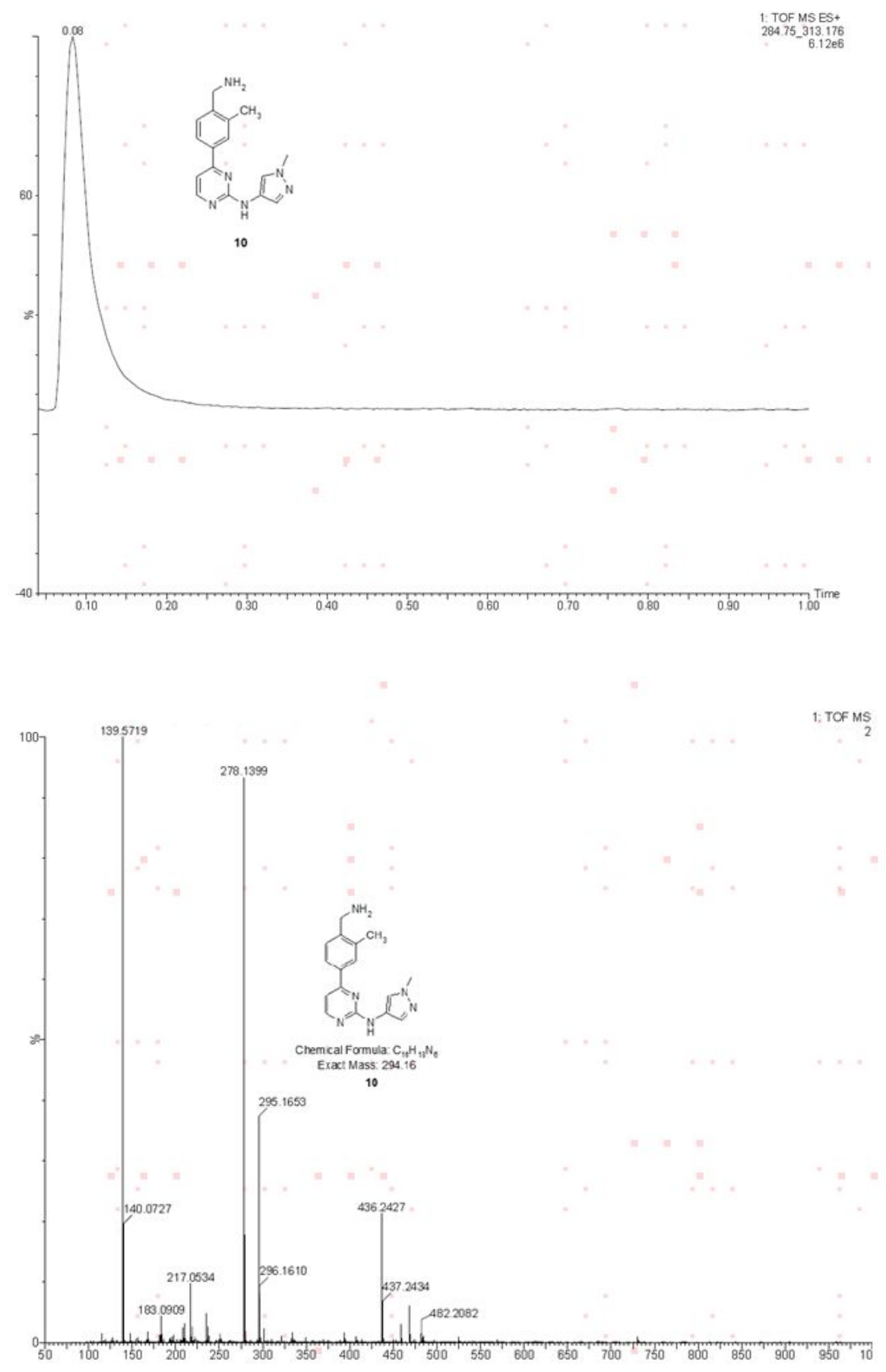

Mo

4 formula(e) evaluated with 1 results within limits (all results (up to 1000) for each mass)

Elements Used:

$\begin{array}{lll}\text { C. } 15-20 & \text { H: } 15-20 & \text { N: } 5-10\end{array}$

1: TOF MS ES*

$7.66 \mathrm{e}+006$

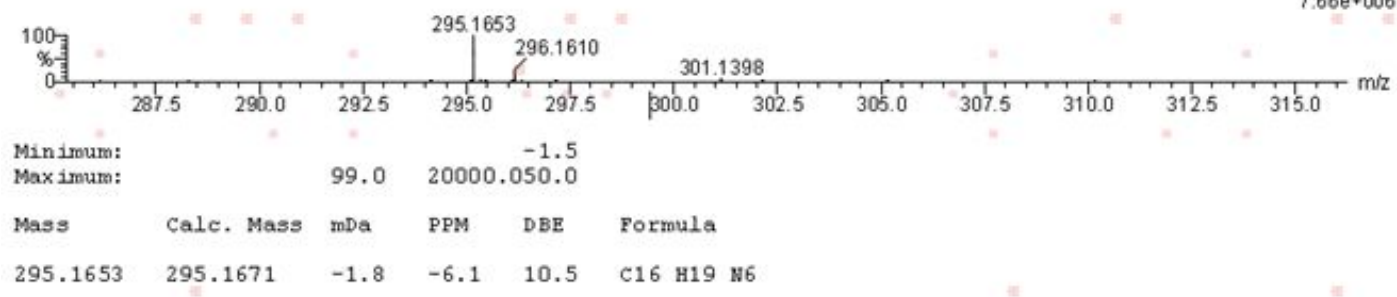


(2) ${ }^{1} \mathrm{HNMR},{ }^{13} \mathrm{CNMR}$, m.p and HRMS spectra of BIIB068 (1)
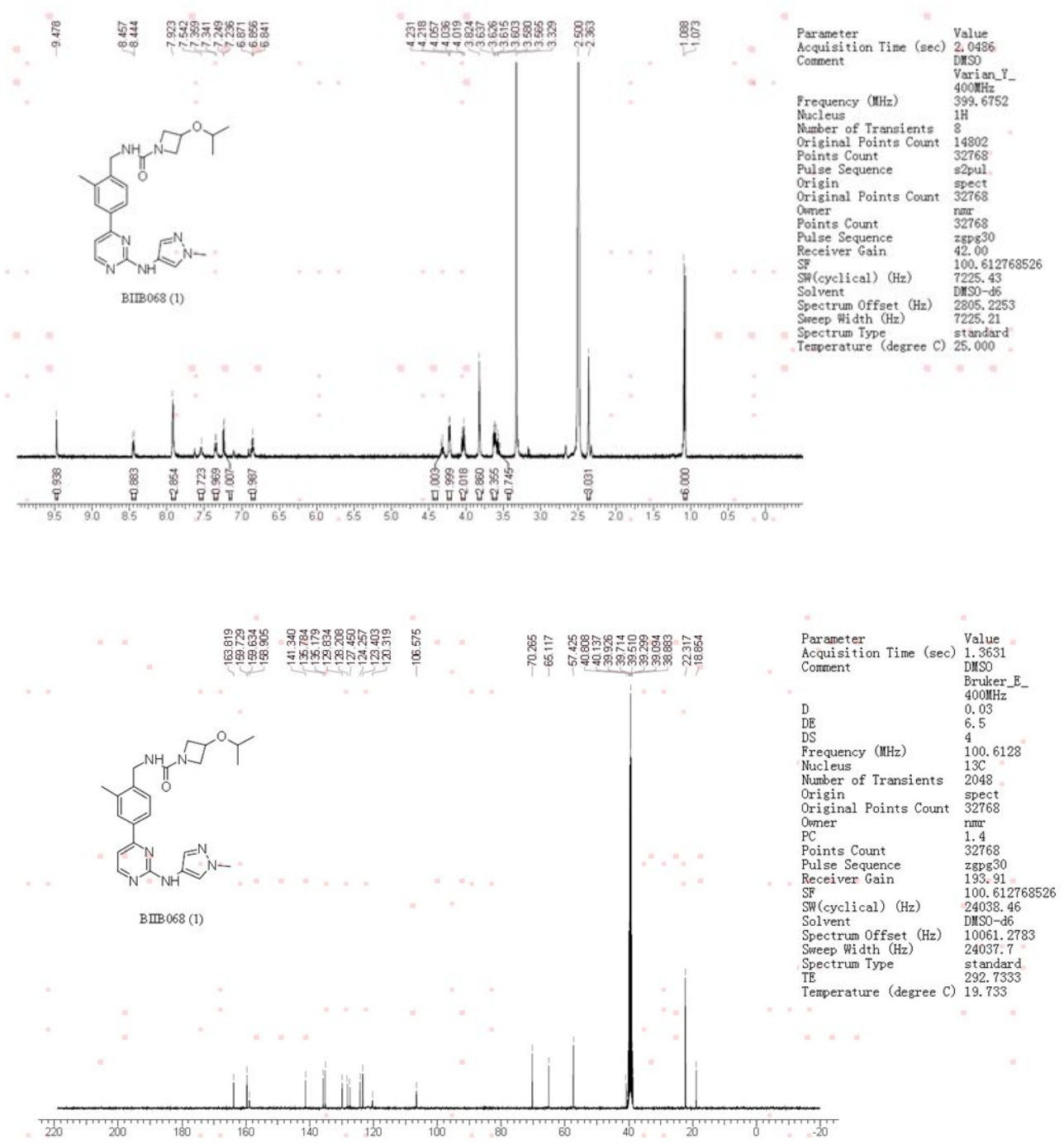

m.p:

Method : ChP2015 Vol. IV 0612(Method I)

Instrument :METTLER MP80 $\left(1.0^{\circ} \mathrm{C} / \mathrm{min}\right)$

Results:

\begin{tabular}{|c|c|c|c|c|}
\hline Iterns & $1\left({ }^{\circ} \mathrm{C}\right)$ & $2\left({ }^{\circ} \mathrm{C}\right)$ & $\beta\left({ }^{\circ} \mathrm{C}\right)$ & $\bar{x}\left({ }^{\circ} \mathrm{C}\right)$ \\
\hline Melting Point & 173.7 & 174.2 & 173.1 & 173.7 \\
\hline
\end{tabular}

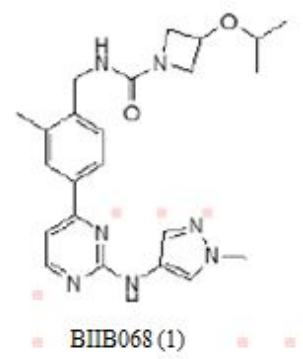


HRMS:
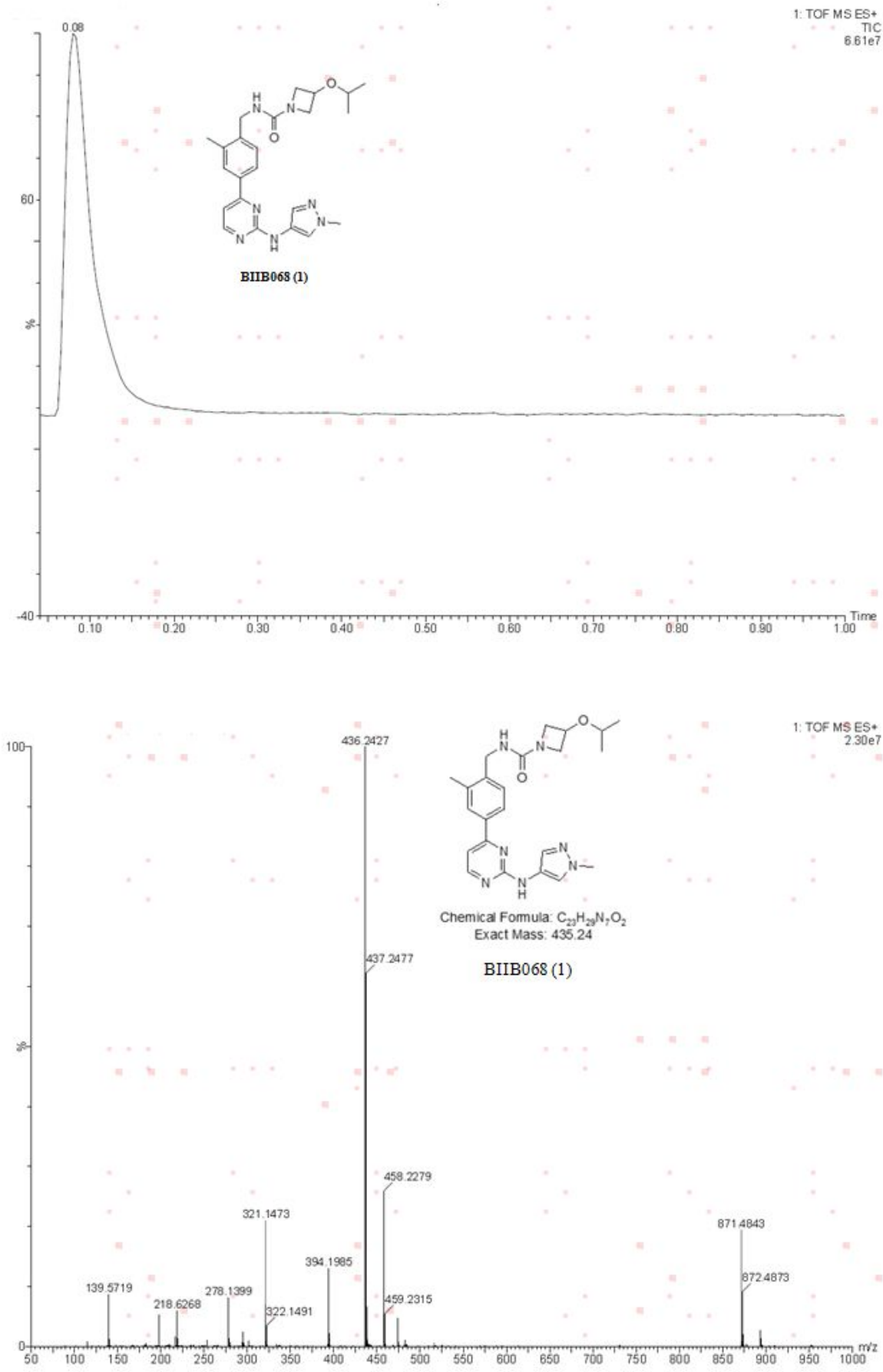

Monoisotopic Mass, Even Eledron lons

6 formula(e) evaluated with 3 results within limits (all results (up to 1000) for each mass)

$\begin{array}{llll}\text { C: } 20-25 & \text { H. } 29-30 & \text { N: } 5-10 & 0: 1-5\end{array}$

1: TOF MS ES+

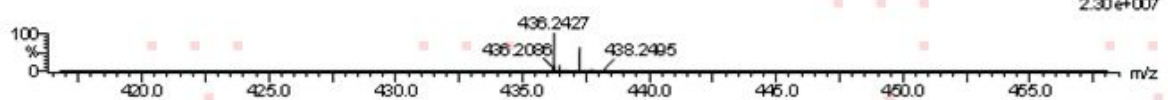

$\begin{array}{ll}\text { Minimian: } & 99.0 \quad 20000.050 .0\end{array}$

Mass Cale. Hass $\mathrm{Da}$ DPM DEB Formula

$\begin{array}{llllllll}436.2427 & 436.2461 & -3.4 & -7.8 & 12.5 & \text { C23 H3O N7 } 02\end{array}$ $\begin{array}{llllll}436.2349 & 7.8 & 17.9 & 12.5 & \text { C24 H3O N5 } 03 \\ 436.2573 & -14.6 & -33.5 & 12.5 & \text { C22 H3O N9 }\end{array}$ 
(2) ${ }^{1} \mathrm{HNMR},{ }^{13} \mathrm{CNMR}$, m.p and HRMS spectra of BIIB068 Hemi-Adipate Salt (19)

${ }^{1} \mathrm{HNMR}$ :

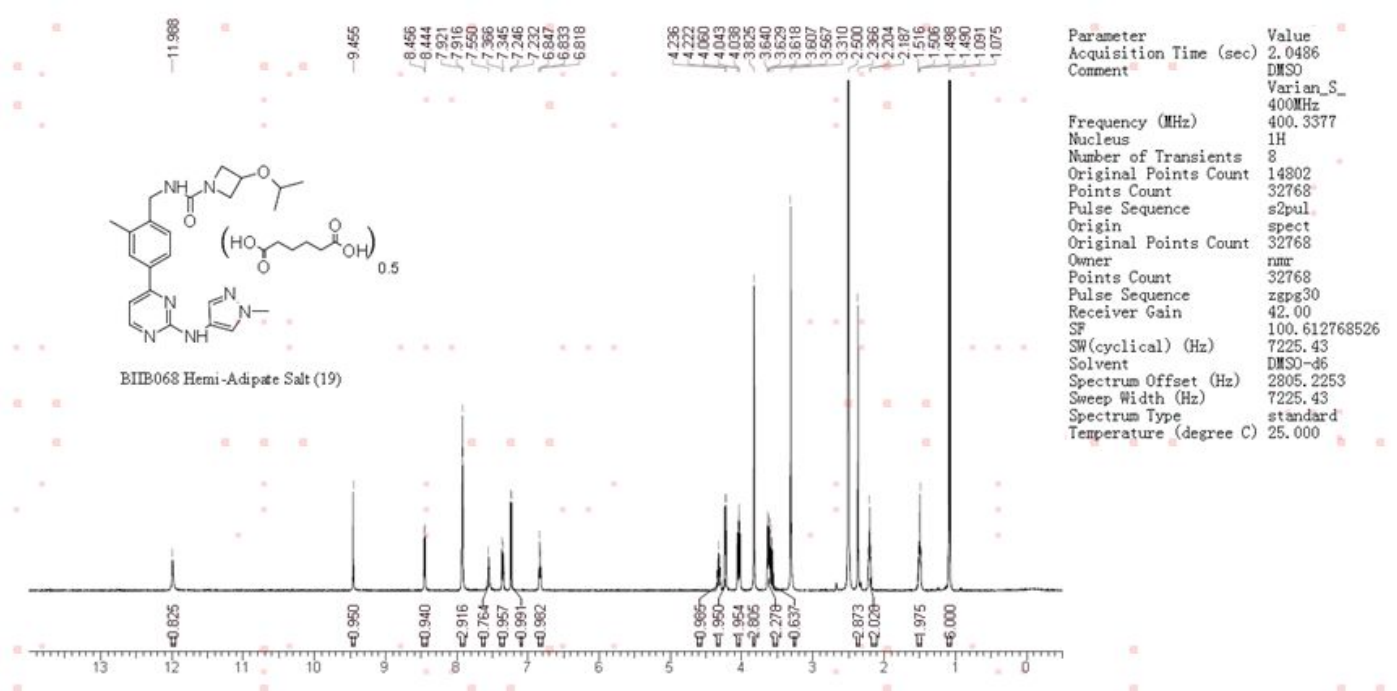

${ }^{13}$ CNMR:

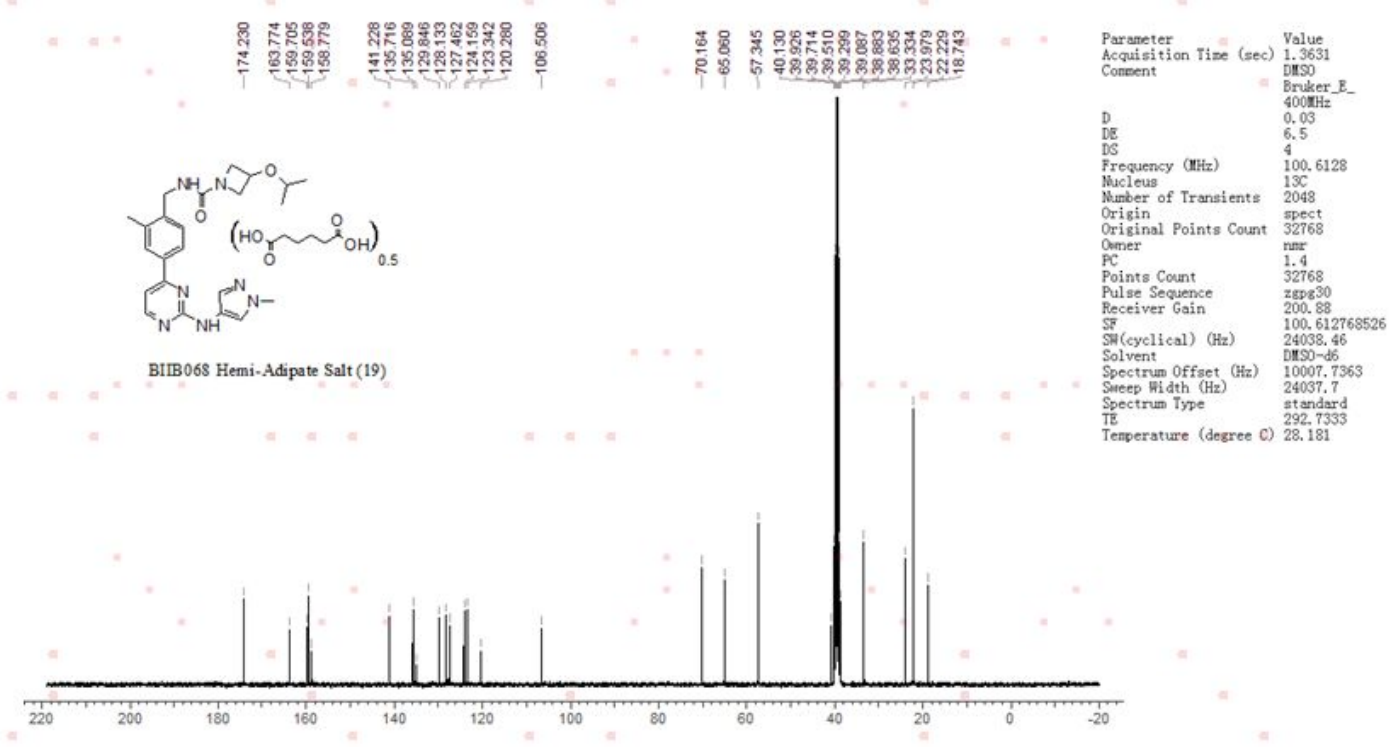

m.p:

Method : ChP2015 Vol. IV 0612 (Method I)

Instrument :METTLER MP80 $\left(1.0^{\circ} \mathrm{C} / \mathrm{min}\right)$

Results:

\begin{tabular}{|c|c|c|c|c|}
\hline Items & $1\left({ }^{\circ} \mathrm{C}\right)$ & $2\left({ }^{\circ} \mathrm{C}\right)$ & $3\left({ }^{\circ} \mathrm{C}\right)$ & $\bar{x}\left({ }^{\circ} \mathrm{C}\right)$ \\
\hline Melting Point & 171.2 & 172.1 & 168.9 & 170.7 \\
\hline
\end{tabular}

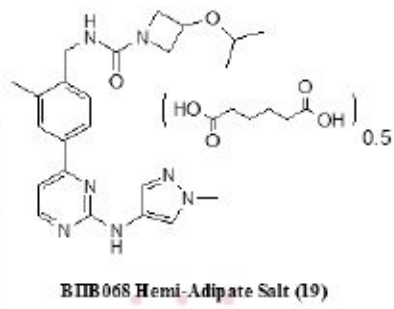


HRMS :
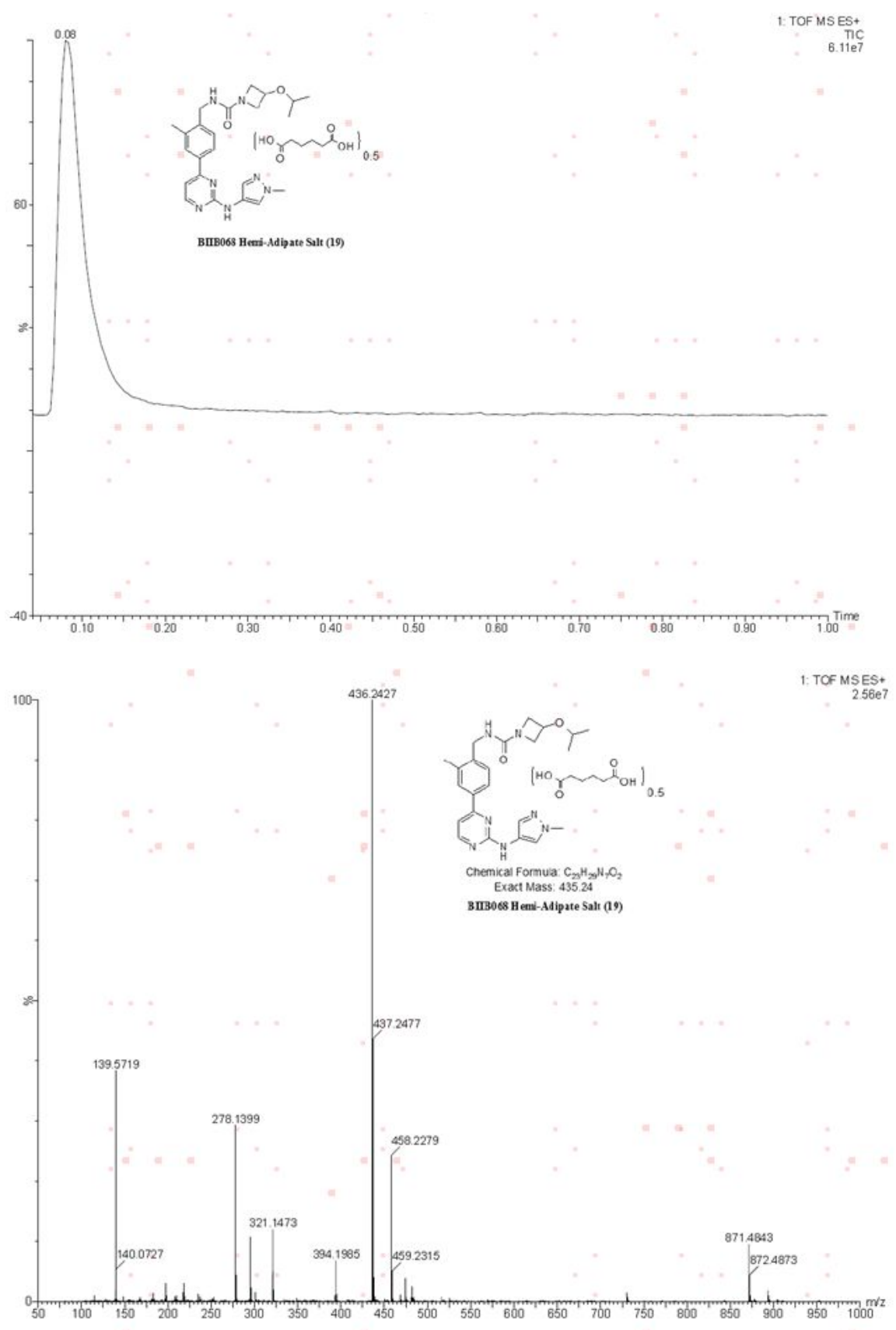

Monoisotopic Mass, Even Electron lons

12 formula(e) evaluated with 6 results within limits (all results (Up to 1000) for each mass)

Elements Used:

$\begin{array}{llll}\text { C: } 20-25 & \text { H: } 25-30 & \text { N: }: 5-10 & 0: 1-5\end{array}$

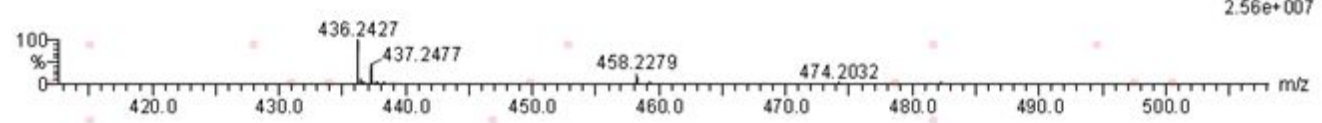

Minimum: $\quad 99.0 \quad 20000.050 .5$

Mass Calc. Mass mDa PPM DBE Formula

$\begin{array}{lllllllll}436.2427 & 436.2461 & -3.4 & -7.8 & 12.5 & \text { C23 } & \text { н30 } & \text { N7 } & \text { O2 }\end{array}$

$\begin{array}{llllll}436.2349 & 7.8 & 17.9 & 12.5 & \text { C24 H30 NS } 03\end{array}$

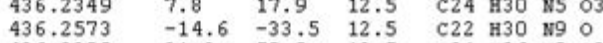

$\begin{array}{llllllll}436.2573 & -14.6 & -33.5 & 12.5 & \text { C22 } & \text { H30 } & \text { N9 } & \circ \\ 436.2209 & 21.8 & 50.0 & 13.5 & \text { C21 } & \text { H26 } & \text { N9 } & \text { O2 }\end{array}$

$\begin{array}{llllllll}436.2209 & 21.8 & 50.0 & 13.5 & \text { C21 } & \text { H26 } & \text { N9 } & 02 \\ 436.2097 & 33.0 & 75.6 & 13.5 & \text { C22 } & \text { H26 } & \text { N7 } & \text { O3 }\end{array}$

$\begin{array}{llllllll}436.2097 & 33.0 & 75.6 & 13.5 & \mathrm{C} 22 & \mathrm{H} 26 & \mathrm{~N} 7 & \mathrm{O} \\ 436.1985 & 44.2 & 101.3 & 13.5 & \mathrm{C} 23 & \mathrm{H} 26 & \mathrm{~N} 5 & \mathrm{O}\end{array}$ 
(4) XRPD report of BIIB068 hemi-adipate salt.

XRPD report

Method Information

\begin{tabular}{|c|c|c|c|}
\hline Method Name & CAS-TJ-GTM-01.00 & Sensing Pattem & Step-measuring \\
\hline X-ray Ggenrator & $\begin{array}{c}\text { Cu, k-Alphal }(\lambda \\
=1.54059 \AA\end{array}$ & Tube Voltage(kV) & 40 \\
\hline Tube Current(mA) & 40 & Divergence Slit(mm) & 1 \\
\hline Primary Soller Slit(mu) & 28 & Secondary Silt(mm) & 28 \\
\hline Detector Slit(mm) & 0.3 & Antiscattering Silt(mm) & 1 \\
\hline Scan axis & 0 s- $\theta \mathrm{d}$ & Step size(deg) & 0.02 \\
\hline Scanning Time(S) & 0.5 & Scanning Scope(deg) & $3-40$ \\
\hline
\end{tabular}

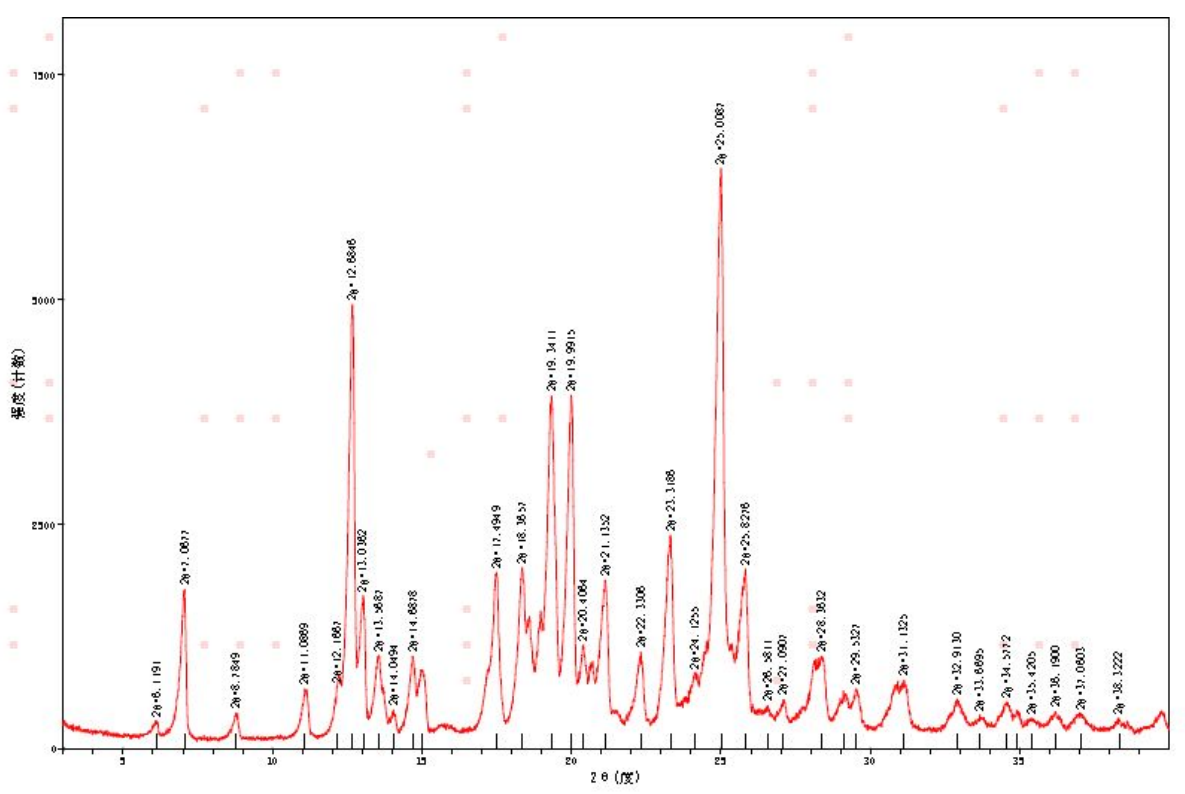

III. Analytical Spectra of Impurities

(1) HPLC, ${ }^{1} \mathrm{HNMR}$ and HRMS spectra of 9 


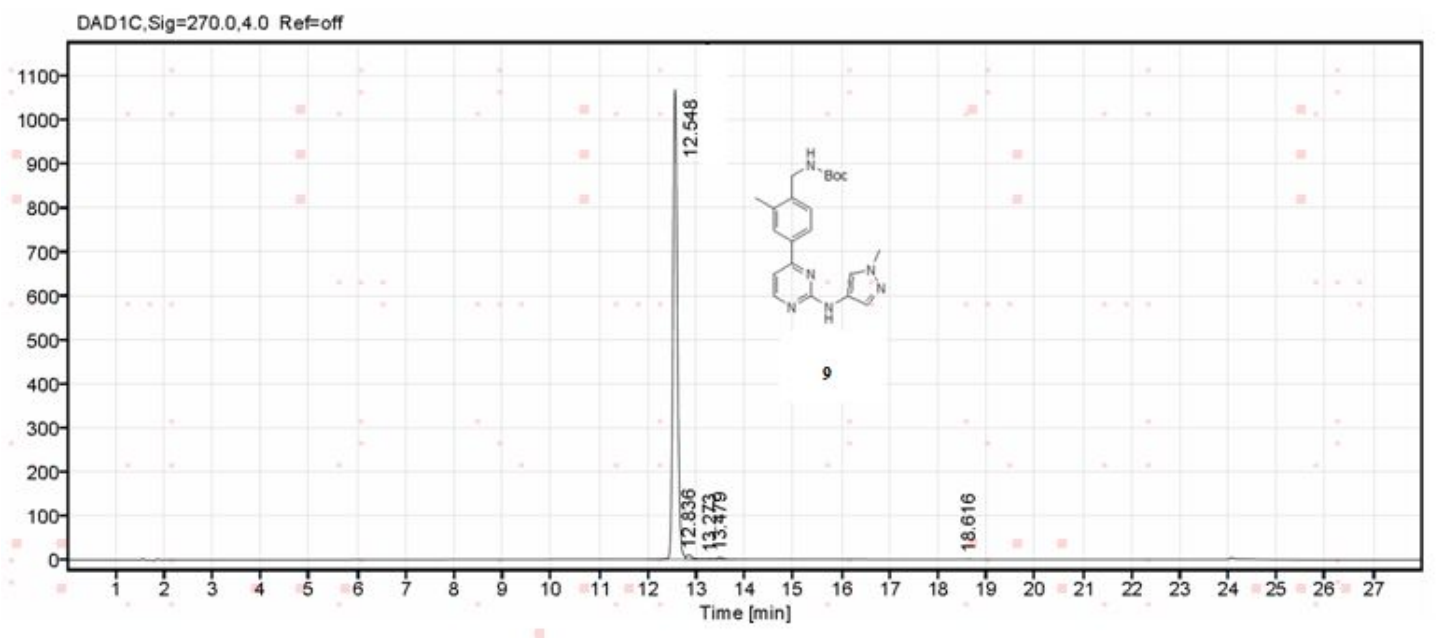

$\begin{array}{cccccc}\text { RT [min] } & \text { RRT } & \text { Resolution USP } & \text { Height (mAU) } & \text { Area } & \text { Area\% } \\ 12.548 & 1.000 & & 1070.5 & 6885.24 & 98.34 \\ 12.836 & 1.023 & 1.5 & 9.9 & 77.40 & 1.11 \\ 13.273 & 1.058 & 1.6 & 0.7 & 7.11 & 0.10 \\ 13.479 & 1.074 & 0.8 & 4.3 & 28.77 & 0.41 \\ 18.616 & 1.484 & 33.1 & 0.6 & 3.18 & 0.05\end{array}$

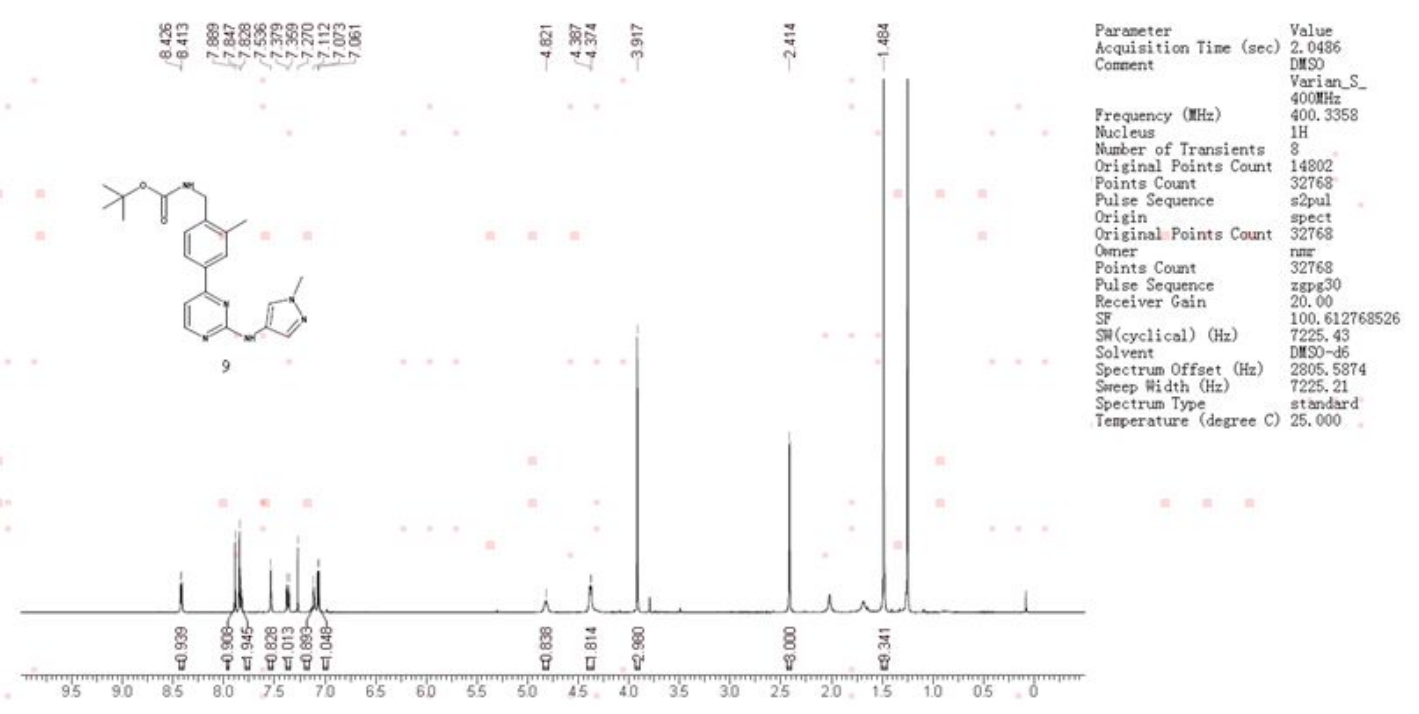

HRMS: 


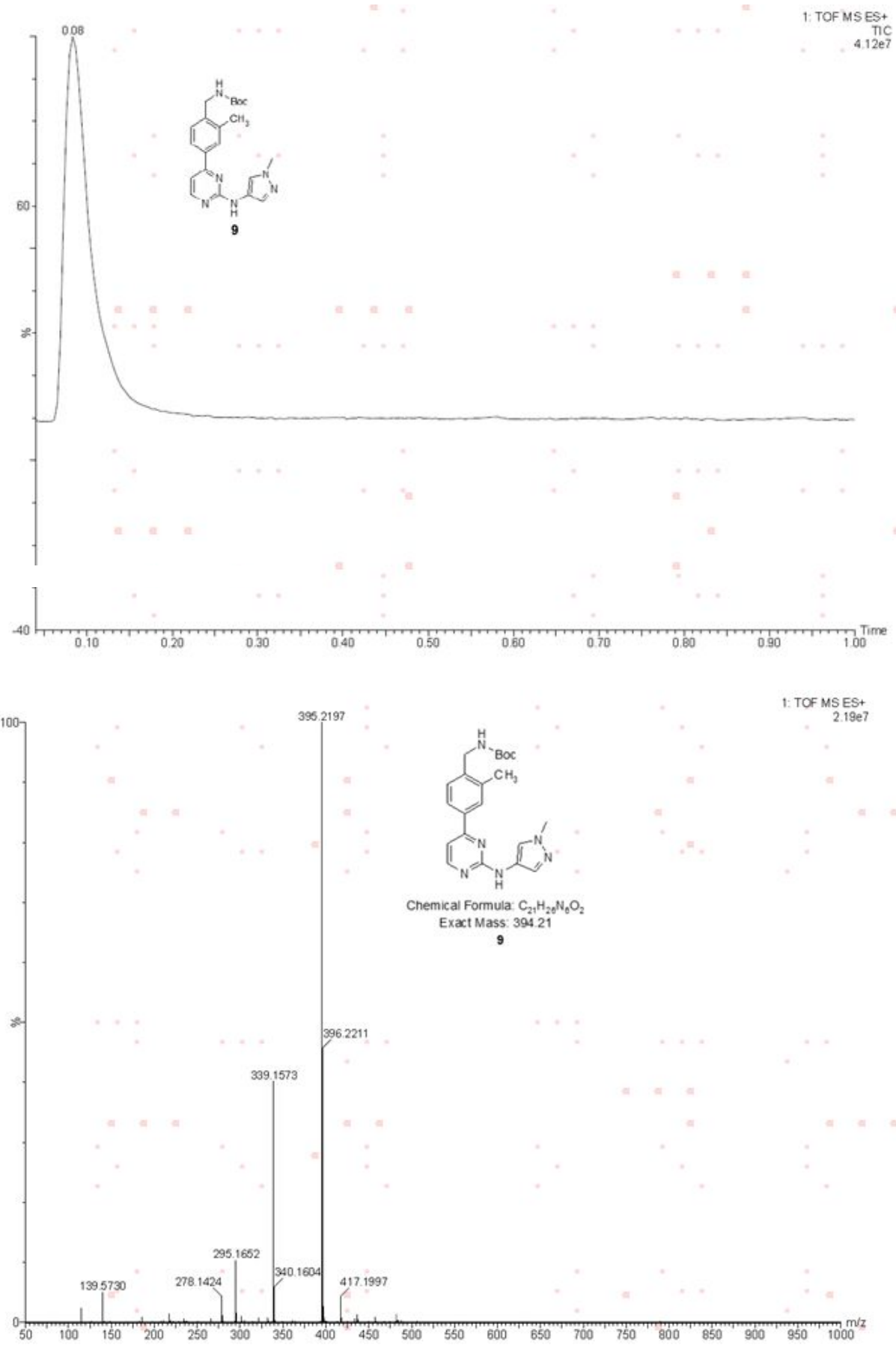

Monoisotopic Mass, Even Eledron Ions

11 formula(e) evaluated with 2 results wthin limits (all results (up to 1000) for each mass)

Elements Used:

$\begin{array}{llll}\text { C: } 20-25 & \text { H. } 25-30 & \text { N. } 5-8 & \text { 0: } 1-5\end{array}$

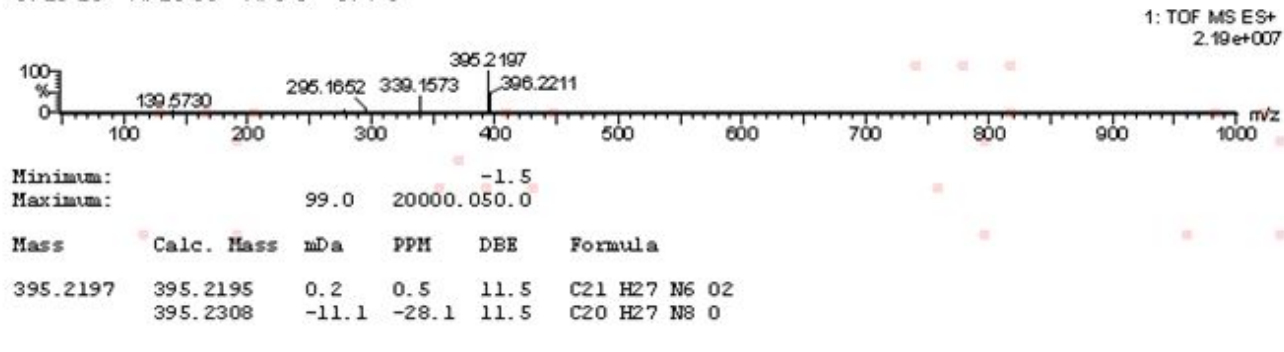


(2) HPLC, ${ }^{1}$ HNMR and HRMS spectra of 12

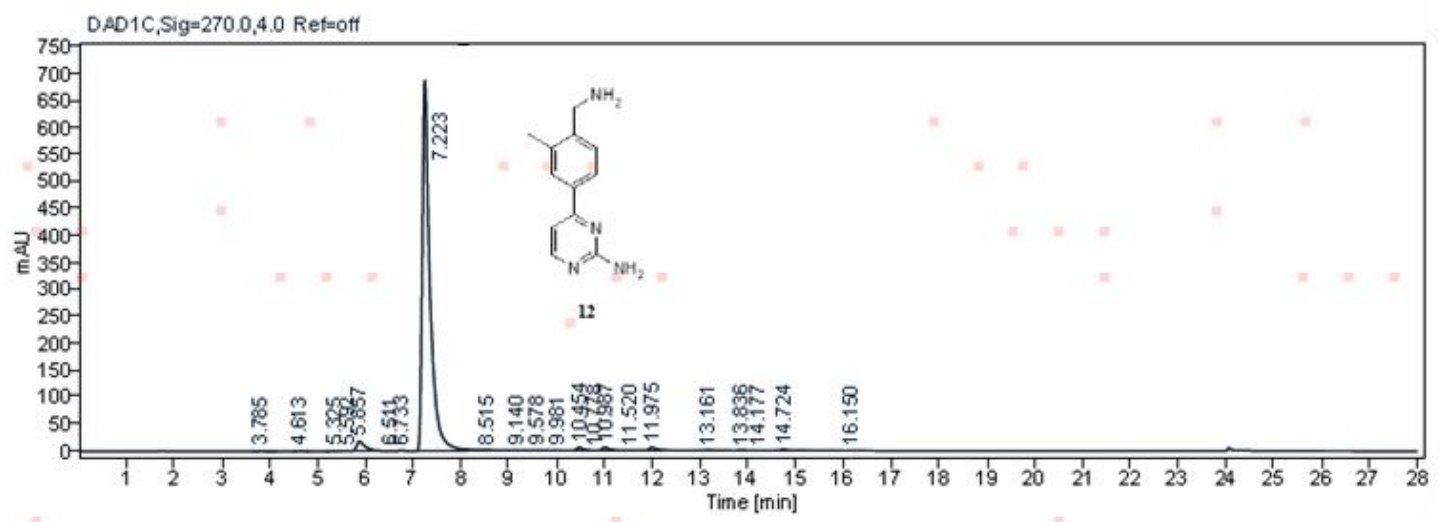

$\begin{array}{cccc}\text { RT [min] } & \text { Height (mAU) } & \text { Area } & \text { Area\% } \\ 6.511 & 0.5 & 4.2 & 0.05 \\ 6.733 & 1.1 & 10.1 & 0.12 \\ 7.223 & 688.2 & 76880 & 98.56 \\ 8.515 & 1.1 & 17.0 & 0.21 \\ 9.140 & 0.2 & 1.9 & 0.02 \\ 9.578 & 0.6 & 5.1 & 0.06 \\ 9.981 & 0.3 & 1.1 & 0.01\end{array}$

${ }^{1} \mathrm{HNMR}$ :

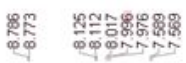

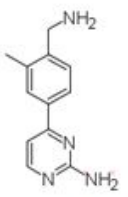

12
롱

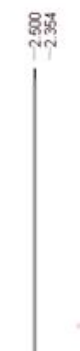

$\begin{array}{ll}\text { Parameter } & \text { Value } \\ \text { Acquisition Time (sec) } 2.0486 \\ \text { Connent }\end{array}$

Prequency $(\mathrm{HHz})$

Nucleus

Original Points Count

Points Count
Pulse Sequence

Origin
Original Points Count 32 spest

Omer
Points Coun

Points Count
Pulse Sequence

Receiver Gain

SW (cyclical) (Hz)

Solvent ${ }_{\text {Spectrum }}$ Offset $(\mathrm{Hz})$

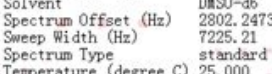

Spectrum Type
Temperature (degree C) 25.000

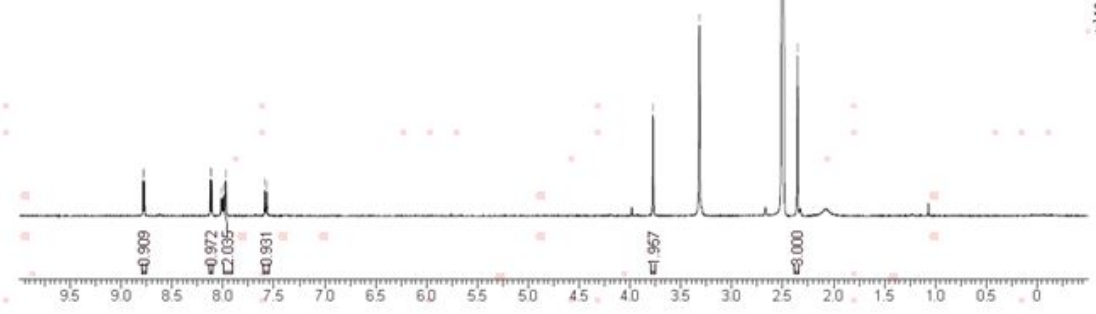

HRMS: 

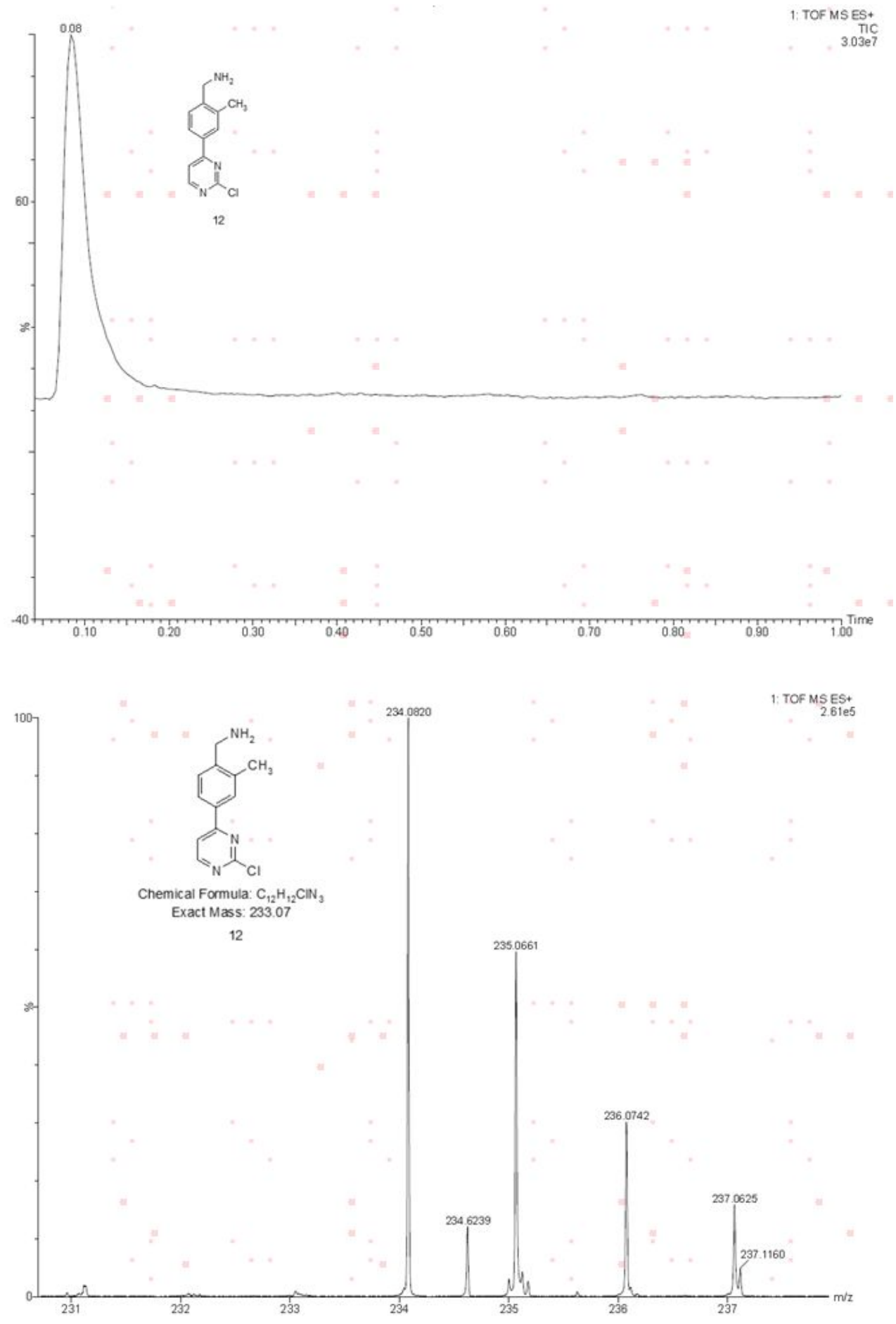

Monoisotopic Mass, Even Electron Ions

7 formula(e) evaluated with 2 results within limits (all results (up to 1000) for each mass)

Elements Used:

C: $10-15 \quad \mathrm{H}: 10-15 \quad \mathrm{~N}: 0-4 \quad \mathrm{Cl}: 0-2$

1: TOF MSES+

$261 \mathrm{e}+005$

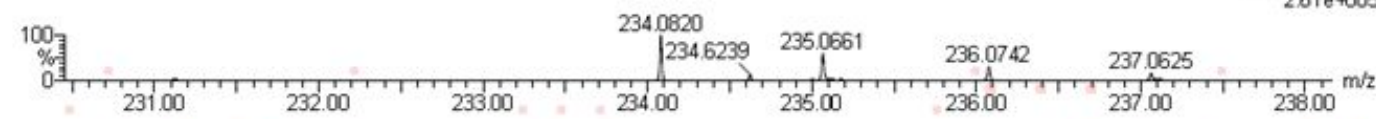

$\begin{array}{lr}\text { Minimur: } & -1.5 \\ \text { Maximum: } & 99.0 \quad 20000.050 .0\end{array}$

Mass Calc. Mass mDa PPM DBE Formula

$\begin{array}{lllllllll}234.0820 & 234.0798 & 2.2 & 9.4 & 7.5 & \text { C12 } & \text { H13 } & \text { N3 } & \text { Cl }\end{array}$

$234.1031 \quad-21.1 \quad-90.1 \quad 11.5 \quad$ C15 H12 $\mathrm{N3}$ 
(3) LCMS spectra of $\mathbf{1 8}$

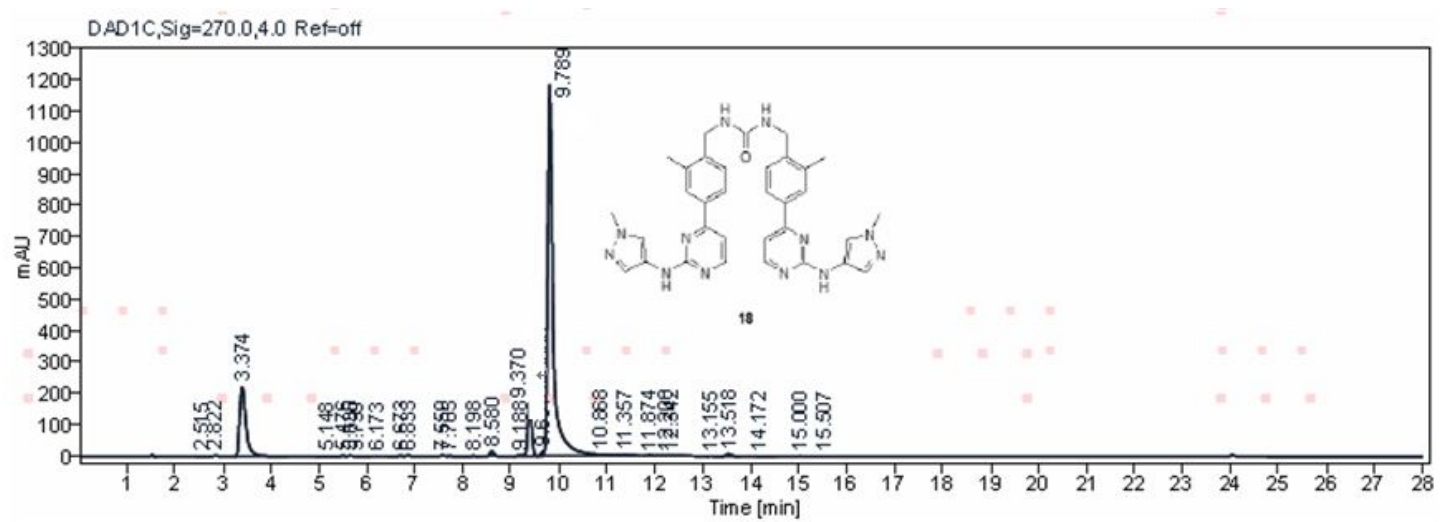

Peak RT 9.863

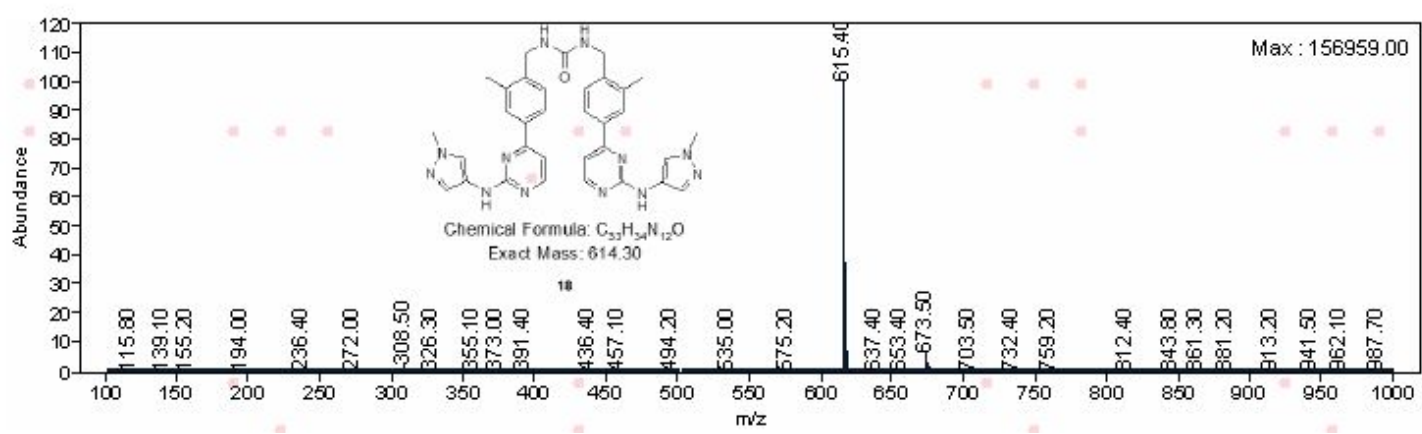

\section{RT [min]}

$\begin{array}{lr}5.758 & 0.4 \\ 5.850 & 0.3 \\ 6.205 & 0.2 \\ 6.675 & 1.5 \\ 6.838 & 2.2 \\ 7.557 & 2.0 \\ 7.717 & 0.9 \\ 8.202 & 0.9 \\ 8.591 & 12.2 \\ 9.202 & 1.9 \\ 9.382 & 147.1 \\ 9.662 & 11.0 \\ 9.798 & 934.9\end{array}$

Area

1.9

1.5

1.2

8.9

10.5

10.8

6.9

4.7

61.8

14.0

724.5

38.9

6382.2
Area\%

0.02

0.02

0.01

0.10

0.12

0.12

0.08

0.05

0.71

0.16

8.36

0.45

73.67

(4) LCMS spectra of $\mathbf{1 5}$ 


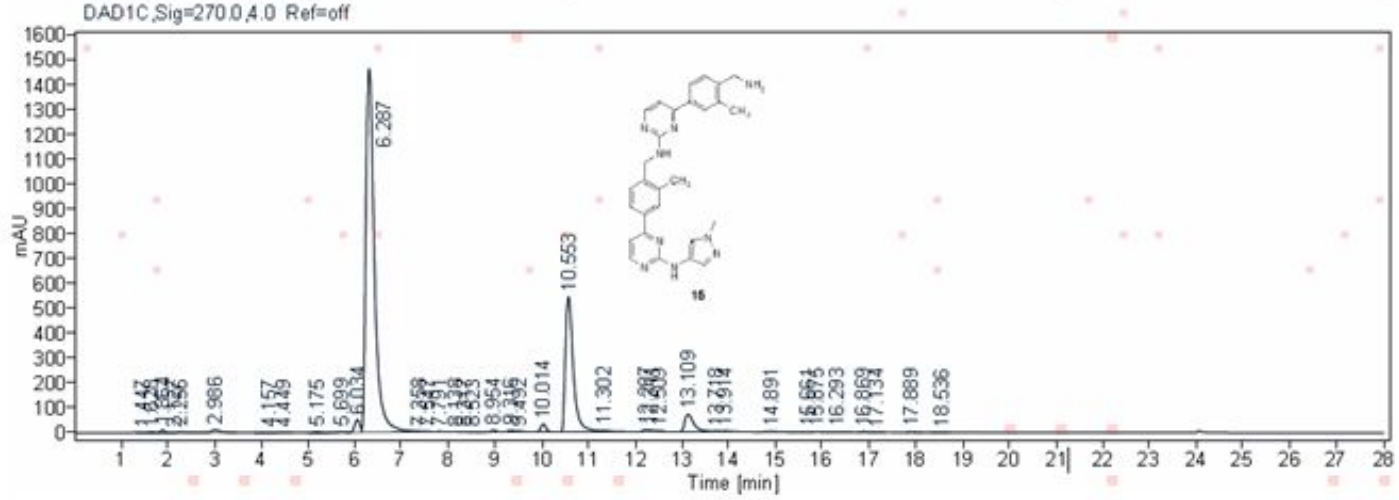

Peak RT $\quad 10.617$

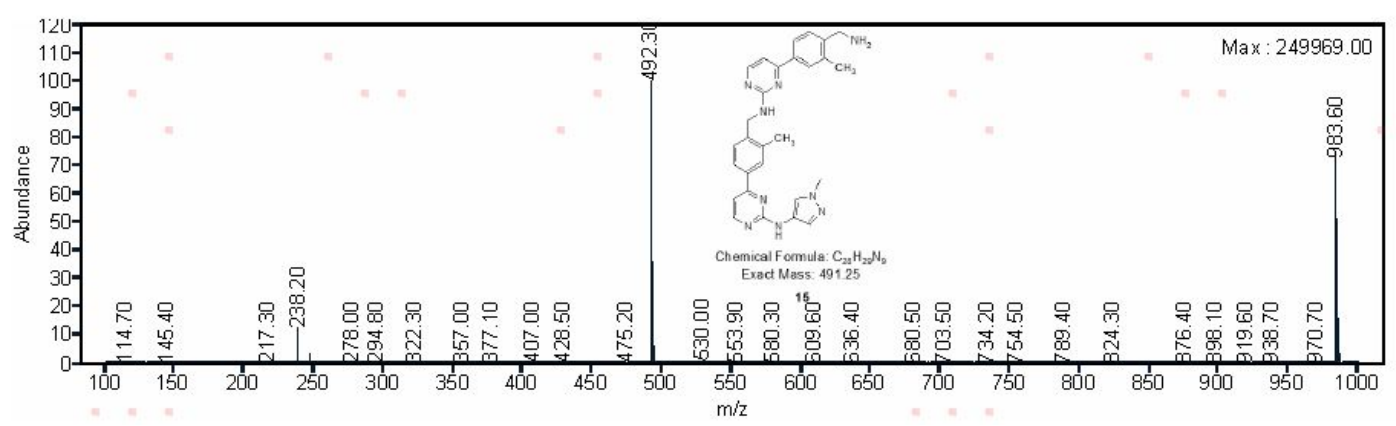

RT [min]

$\begin{array}{lc}2.986 & 11.1 \\ 4.157 & 0.3 \\ 4.449 & 0.4 \\ 5.175 & 2.6 \\ 5.699 & 3.0 \\ 6.034 & 51.6 \\ 6.287 & 1463.7 \\ 7.358 & 2.0 \\ 7.547 & 0.8 \\ 7.791 & 1.1 \\ 8.138 & 1.3 \\ 8.317 & 1.5 \\ 8.523 & 0.4 \\ 8.954 & 6.5 \\ 9.316 & 5.4 \\ 9.492 & 3.2 \\ 10.014 & 31.6 \\ 10.553 & 543.5\end{array}$

\section{Area}

139.0

5.6

3.8

25.5

25.5

385.4

18677.0

11.9

5.1

10.4

13.0

12.3

2.9

58.1

53.2

33.6

243.0

6437.2
A rea\%

0.50

0.02

0.01

0.09

0.09

1.40

67.74

0.04

0.02

0.04

0.05

0.04

0.01

0.21

0.19

0.12

0.88

23.35 
IV. Analytical Spectra of Reaction Optimization (7 to 10, table 1)

Crude HPLC of Table 1, entries 1-15 are shown below:

Entry 1 HPLC spectra

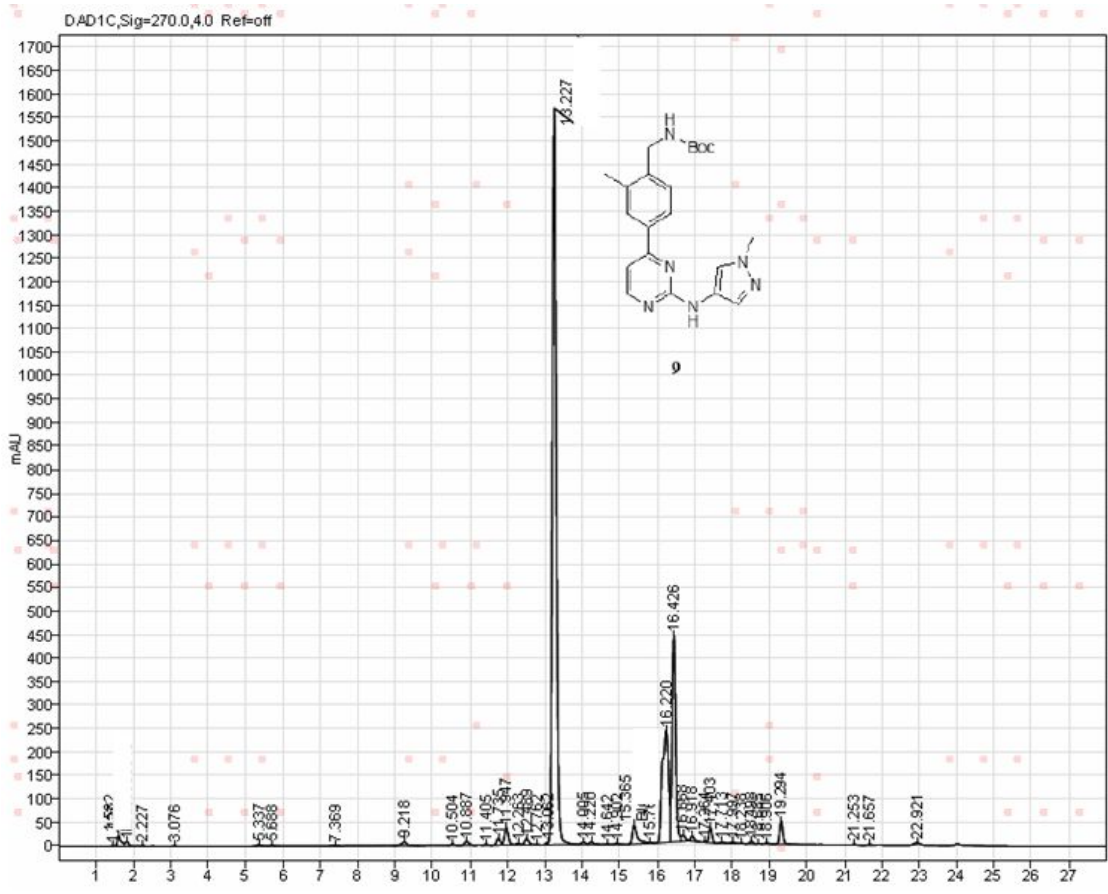

\begin{tabular}{|c|c|c|c|c|c|c|}
\hline RT [min] & & & Area & Area\% & Resolution USP & Height(msu \\
\hline 1.808 & & & 71.23 & 0.37 & 13 & 10.3 \\
\hline 2.227 & & & 5.90 & 003 & 25 & 12 \\
\hline 3.076 & & & 4.97 & 003 & 53 & 0.7 \\
\hline 5.337 & & & 28.93 & 0.15 & 10.7 & 32 \\
\hline 5.688 & & & 11.79 & 006 & 16 & 1.5 \\
\hline 7.369 & & $=$ & 4.87 & 003 & 73 & 0.5 \\
\hline 9.218 & $=$ & & 75.33 & 0.39 & 78 & 80 \\
\hline 10504 & & & 18.21 & 009 & 6.6 & 2.6 \\
\hline 10887 & & & 69.58 & 0.36 & 20 & 8.4 \\
\hline 11.405 & & & 12.46 & 006 & 2.7 & 20 \\
\hline 11.735 & & & 10067 & 0.52 & 18 & 14.2 \\
\hline 11947 & & & 270.48 & 1.40 & 1.1 & 37.4 \\
\hline 12263 & & & 27.78 & 0.14 & 1.6 & 36 \\
\hline 12.489 & & & 10639 & 0.55 & 1.1 & 13.5 \\
\hline 12.762 & & & 8.48 & 004 & 12 & 12 \\
\hline 13062 & & & 22.02 & 0.11 & & 5.5 \\
\hline 13227 & & & 1128797 & 58.39 & & 1567.8 \\
\hline 14005 & & & 40.00 & 021 & 38 & 5.1 \\
\hline 14220 & & & 52.31 & 027 & 1.1 & 59 \\
\hline 14842 & $=$ & $=$ & 7.75 & 004 & 22 & 12 \\
\hline 14902 & & & 24.64 & 0.13 & 1.5 & 3.4 \\
\hline 15365 & & & 37820 & 196 & 2.4 & 40.7 \\
\hline
\end{tabular}


Entry 2 HPLC spectra

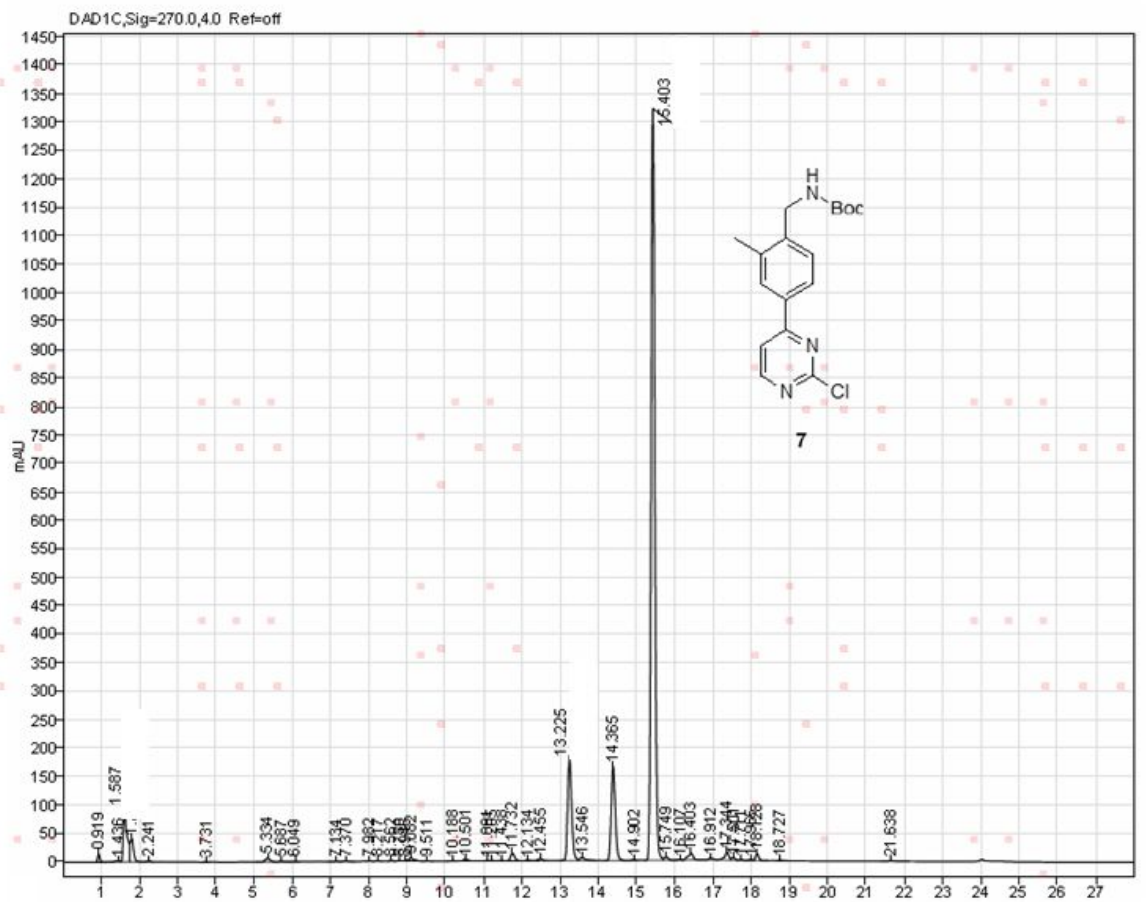

\begin{tabular}{|c|c|c|c|c|c|c|c|c|}
\hline RT [min] & & Area & Area\% & & & Resolution USP & & Height(mAU) \\
\hline 1.587 & & 640.74 & 4.82 & & & 0.7 & & 93.1 \\
\hline 1.785 & & 259.89 & 1.96 & & & 0.9 & & 40.9 \\
\hline 2.241 & $=$ & 10.74 & 0.08 & & $=$ & 2.1 & & 1.5 \\
\hline 3.731 & & 4.30 & 0.03 & & " & 7.9 & & $=0.6$ \\
\hline 5.334 & $=$ & 60.13 & 0.45 & = & & 7.2 & & 6.6 \\
\hline 5.687 & & 9.45 & 0.07 & & & 1.6 & & 1.1 \\
\hline 6.049 & & 5.54 & 0.04 & & & 1.7 & & 0.6 \\
\hline 7.134 & & 4.80 & 0.04 & & & 5.2 & & 0.7 \\
\hline 7.370 & & 10.78 & 0.08 & & $=$ & 1.2 & & $=1.2$ \\
\hline 7.982 & & 5.69 & 0.04 & " & & 2.8 & & 0.8 \\
\hline 8.217 & & 8.10 & 0.06 & & & 1.2 & & 1.0 \\
\hline 8.562 & & 10.79 & 0.08 & & & 2.1 & & 2.1 \\
\hline 8.780 & & 7.53 & 0.06 & & & 1.3 & & 1.1 \\
\hline 8.946 & & 10.39 & 0.08 & & & 0.8 & & 2.0 \\
\hline 9.082 & & 53.46 & 0.40 & & & 0.7 & & 5.9 \\
\hline 9.511 & & 4.31 & 0.03 & & & 2.1 & & 0.6 \\
\hline 10.188 & & 8.66 & 0.07 & " & " & 2.8 & & 0.9 \\
\hline 10.501 & " & 28.19 & 0.21 & & & 1.4 & & 4.0 \\
\hline 11.091 & & 5.21 & $r^{-1}$ & & & 3.0 & & 0.9 \\
\hline 11.205 & & 4.24 & 13 & & & 0.5 & & 0.7 \\
\hline 11.438 & & 6.50 & 15 & & & 0.9 & & 0.8 \\
\hline 11.732 & $=$ & 99.22 & 0.75 & & 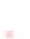 & 1.4 & & 13.3 \\
\hline 12.134 & & 10.86 & 0.08 & & $=$ & 1.7 & & $=1.0$ \\
\hline 12.455 & $=$ & 31.37 & 0.24 & $=$ & & 1.3 & & 3.8 \\
\hline 13.225 & & 1289.20 & 9.70 & & & 3.9 & & 176.6 \\
\hline 13.546 & & 52.88 & 0.40 & & & 1.3 & & 4.6 \\
\hline 14.365 & & 1234.36 & 9.29 & & & 3.3 & & 165.8 \\
\hline 14.902 & $=$ & 9.12 & 0.07 & & $=$ & 3.0 & $=$ & 1.5 \\
\hline 15.403 & & 8893.35 & 66.92 & & " & 2.9 & & " 1321.2 \\
\hline
\end{tabular}


Entry 3 HPLC spectra

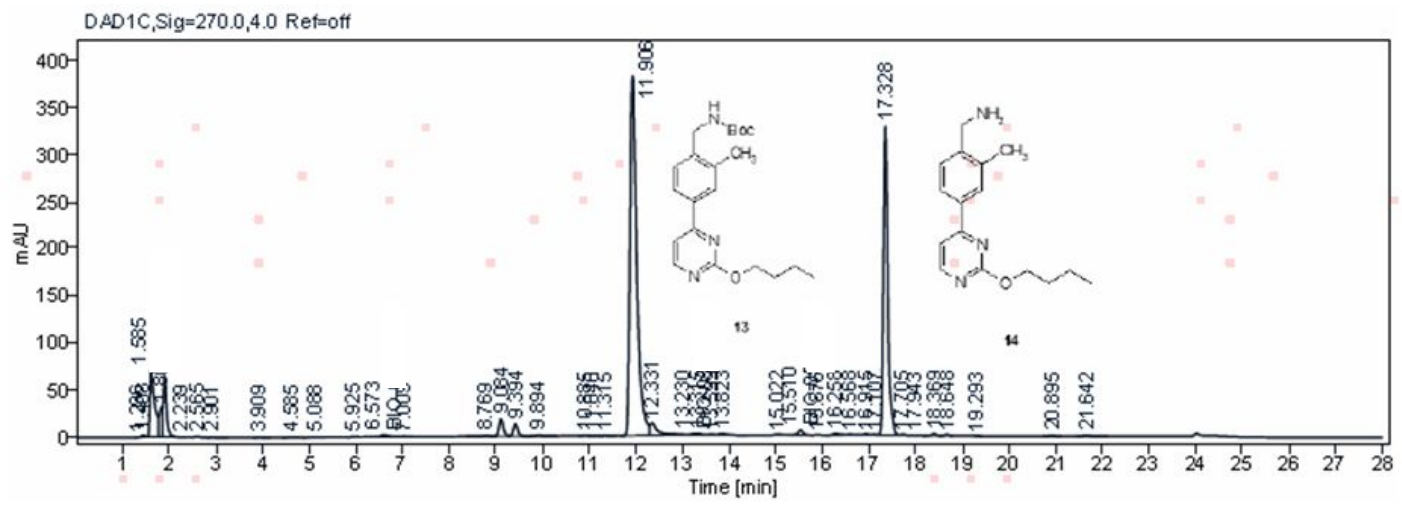

\begin{tabular}{|c|c|c|c|c|}
\hline $\mathbf{R T}$ [min] & & Height (mAU) & Area & Area $\%$ \\
\hline 2.239 & & 0.2 & 0.8 & 0.01 \\
\hline 2.565 & & $=0.9$ & 8.4 & 0.11 \\
\hline 2.901 & " & 0.1 & 1.1 & 0.01 \\
\hline 3.909 & & 0.2 & 1.8 & 0.02 \\
\hline 4.585 & & 0.4 & 4.2 & 0.06 \\
\hline 5.088 & & 0.4 & 3.4 & 0.05 \\
\hline 5.925 & & 0.3 & 2.6 & 0.03 \\
\hline 6.573 & 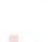 & 2.1 & 18.5 & 0.24 \\
\hline 7.009 & & 0.2 & 1.9 & 0.02 \\
\hline 8.769 & & 0.6 & 5.2 & 0.07 \\
\hline 9.084 & & 18.1 & 120.0 & 1.58 \\
\hline 9.394 & & 13.1 & 86.2 & 1.14 \\
\hline 9.894 & & 1.1 & 11.1 & 0.15 \\
\hline 10.885 & & 0.4 & 3.5 & 0.05 \\
\hline 11.040 & & 0.3 & 2.8 & 0.04 \\
\hline 11.315 & & $=0.3$ & $2.8=$ & 0.04 \\
\hline 11.906 & $=$ & 382.6 & 3875.9 & 51.11 \\
\hline 12.331 & & 12.8 & 156.1 & 2.06 \\
\hline 13.230 & & 1.2 & 6.1 & 0.08 \\
\hline 13.315 & & 1.4 & 9.7 & 0.13 \\
\hline 13.599 & & 0.6 & 4.5 & 0.06 \\
\hline 13.823 & . & 1.4 & 14.6 & 0.19 \\
\hline 15.022 & & 1.1 & 11.6 & 0.15 \\
\hline 15.510 & & 5.5 & 44.4 & 0.59 \\
\hline 15.876 & & 0.3 & 2.4 & 0.03 \\
\hline 16.258 & & 1.7 & 18.1 & 0.24 \\
\hline 16.568 & & 0.6 & 4.9 & 0.06 \\
\hline 16.915 & " & 1.0 & 7.2 & 0.10 \\
\hline 17.107 & & 0.8 & 5.1 & 0.07 \\
\hline 17.328 & & 329.0 & 2156.5 & \\
\hline
\end{tabular}


Entry 4 HPLC spectra

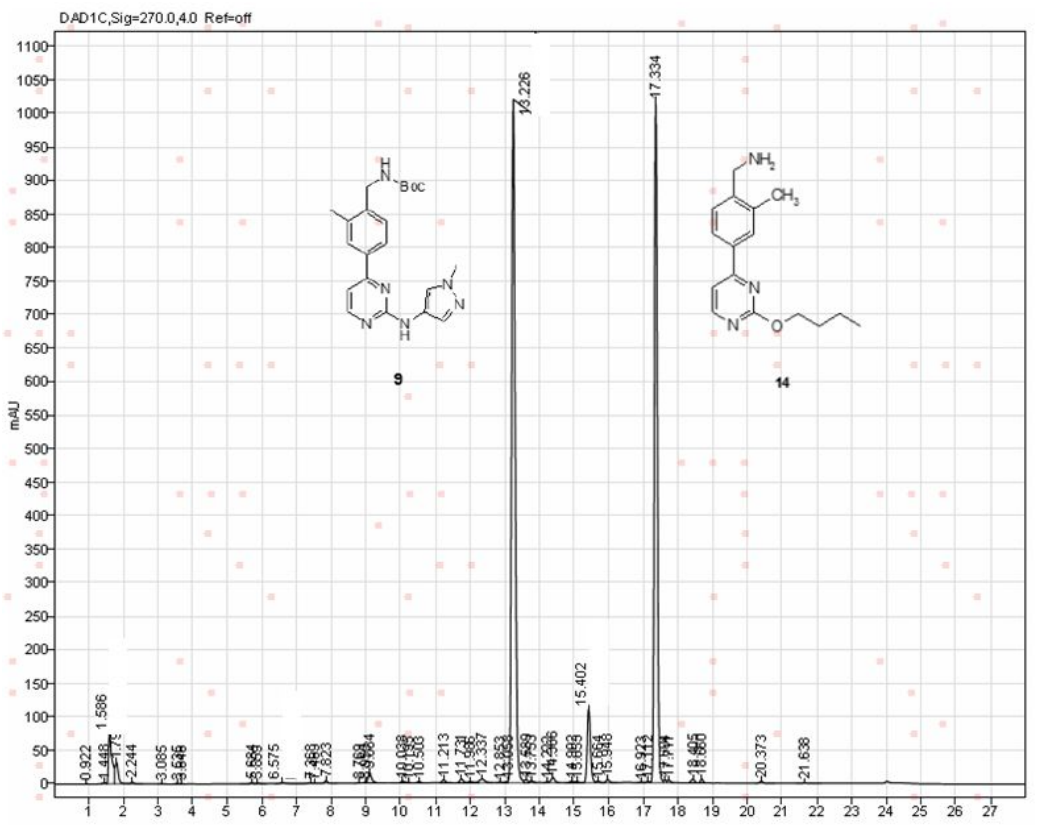

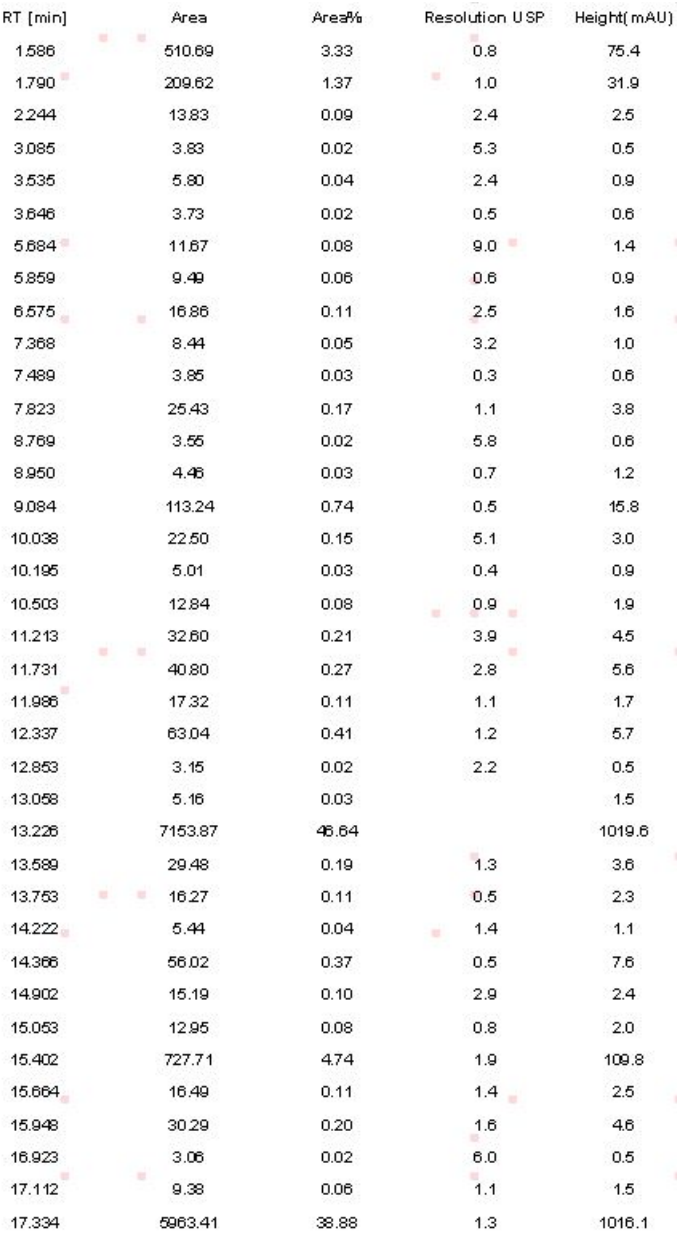


Entry 5 HPLC spectra

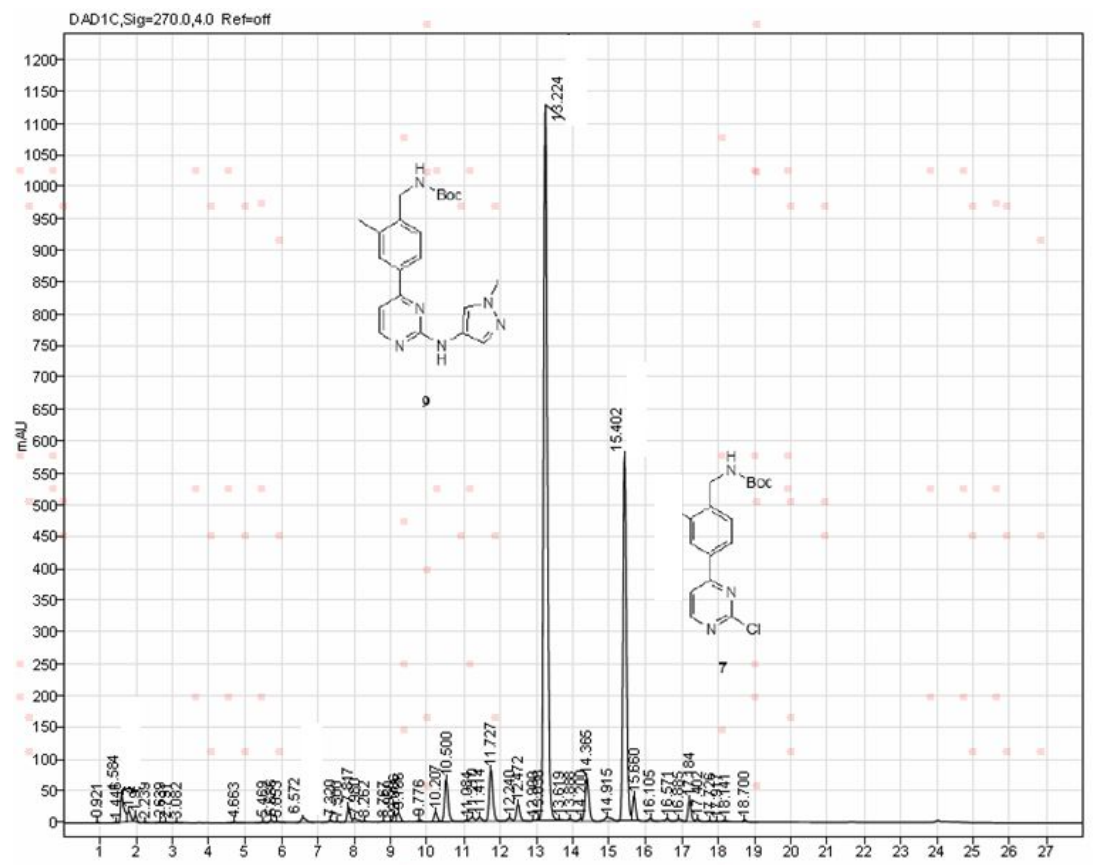

RT [min]

1.584

1.788

1.965

2.239

2.639

2.791

3.082

4.663

12.240

12.472

12.900

13.038

13.224

15.402

15.660

16.105
Area

285.29

98.40

43.88

2.77

8.07

7.21

7.01

11.41

36.73

189.43

2.88

11.33

7980.11

3799.15

243.55

27.29
Area\%

1.84

0.64

0.28

0.02

0.05

0.05

0.05

0.07

0.24

1.22

0.02

0.07

51.55

24.54

1.57

0.18
Resolution USP

Height(mAU)

43.7

16.0

10.0

0.6

1.0

0.8

0.9

1.0

7.1

2.6

4.7

26.4

0.7

2.8

$0.9 \quad 1126.3$

1.6

574.2

1.5

39.3

2.7

4.3 
Entry 6 HPLC spectra

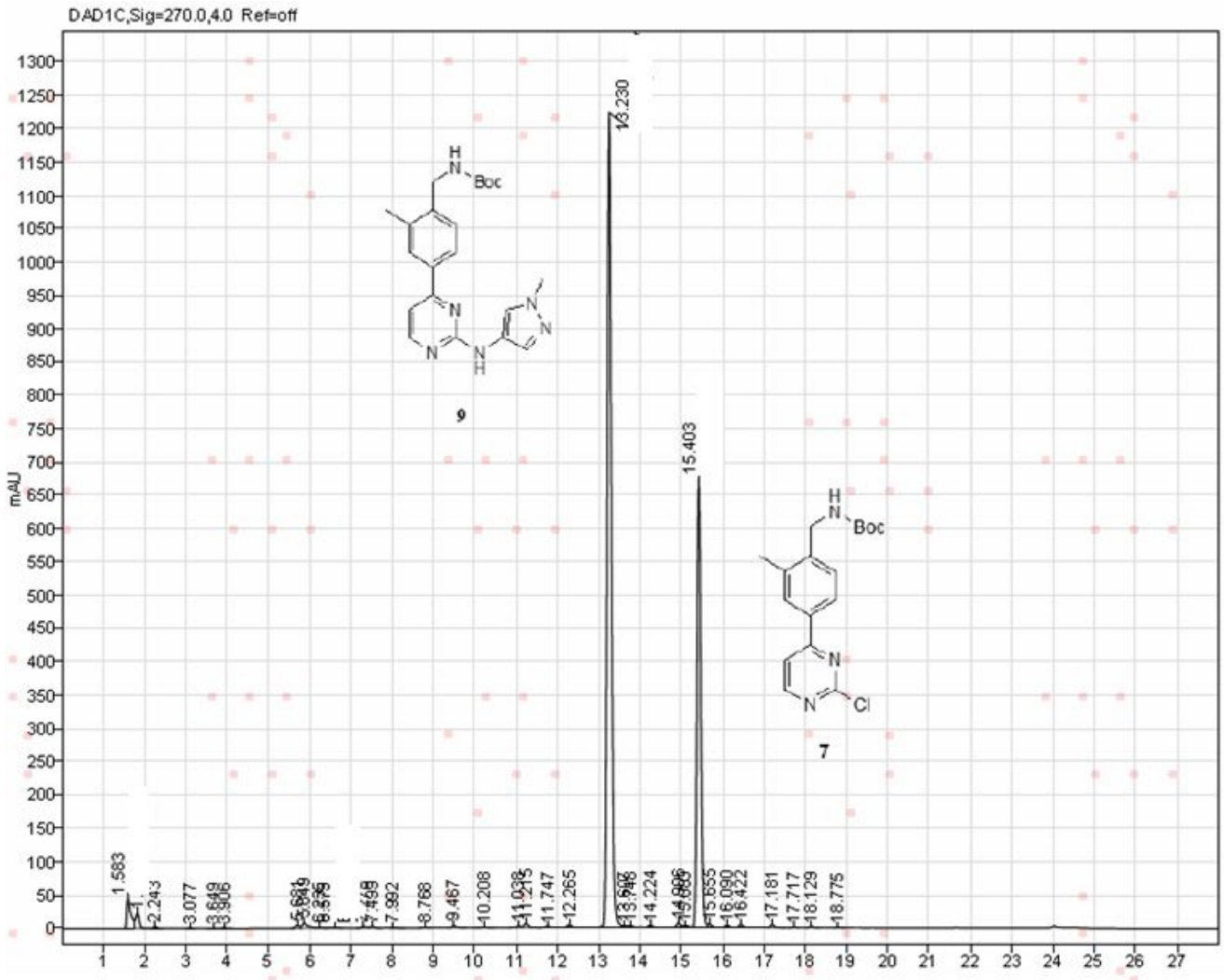

\begin{tabular}{|c|c|c|c|c|c|c|c|c|}
\hline RT [min] & " & Area & Area $\%$ & & & Resolution USP & & Height(mAU) \\
\hline 2.243 & & 14.43 & 0.10 & & . & 2.8 & & 2.8 \\
\hline 3.077 & & 5.65 & 0.04 & & & 5.6 & & 0.9 \\
\hline 3.649 & & 5.49 & 0.04 & & & 3.0 & & 0.6 \\
\hline 3.906 & & 11.50 & 0.08 & & & 1.1 & & 1.1 \\
\hline 5.681 & & 29.33 & 0.21 & & 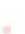 & 7.7 & & 4.3 \\
\hline 5.849 & . & 72.53 & 0.51 & & . & 0.8 & & 7.6 \\
\hline 6.235 & & 7.32 & 0.05 & $=$ & & 1.7 & & 0.8 \\
\hline 6.575 & & 8.87 & 0.06 & & & 1.1 & & 0.7 \\
\hline 7.278 & & 21.71 & 0.15 & & & 2.2 & & 2.3 \\
\hline 7.499 & & 18.41 & 0.13 & & & 0.9 & & 2.4 \\
\hline 7.992 & & 5.31 & 0.04 & & & 2.0 & & 0.5 \\
\hline 8.768 & & 4.88 & 0.03 & & & 3.3 & & 0.6 \\
\hline 9.467 & & 21.58 & 0.15 & & & 3.7 & & 2.9 \\
\hline 10.208 & & 7.36 & 0.05 & " & $=$ & 4.1 & & 0.9 \\
\hline 11.038 & " & 16.00 & 0.11 & & & 4.4 & $=$ & 2.1 \\
\hline 11.215 & & 41.80 & 0.30 & & & 1.0 & & 6.0 \\
\hline 11.747 & & 13.43 & 0.10 & & & 2.6 & & 1.6 \\
\hline 12.265 & & 28.81 & 0.20 & & & 2.5 & & 4.0 \\
\hline 13.230 & $=$ & 8655.97 & 61.36 & & . & 5.2 & & 1223.1 \\
\hline 13.607 & & 13.17 & 0.09 & & $=$ & 0.7 & $=$ & $=2.2$ \\
\hline 13.748 & & 15.54 & 0.11 & $=$ & & 0.2 & & 2.1 \\
\hline 14.224 & & 23.08 & 0.16 & & & 2.0 & & 3.1 \\
\hline 14.906 & & 40.96 & 0.29 & & & 3.9 & & 6.4 \\
\hline 15.083 & & 20.08 & 0.14 & & & 0.8 & & 2.2 \\
\hline 15.403 & & 4429.56 & 31.40 & & $=$ & 14 & & 674.4 \\
\hline
\end{tabular}


Entry 7 HPLC spectra

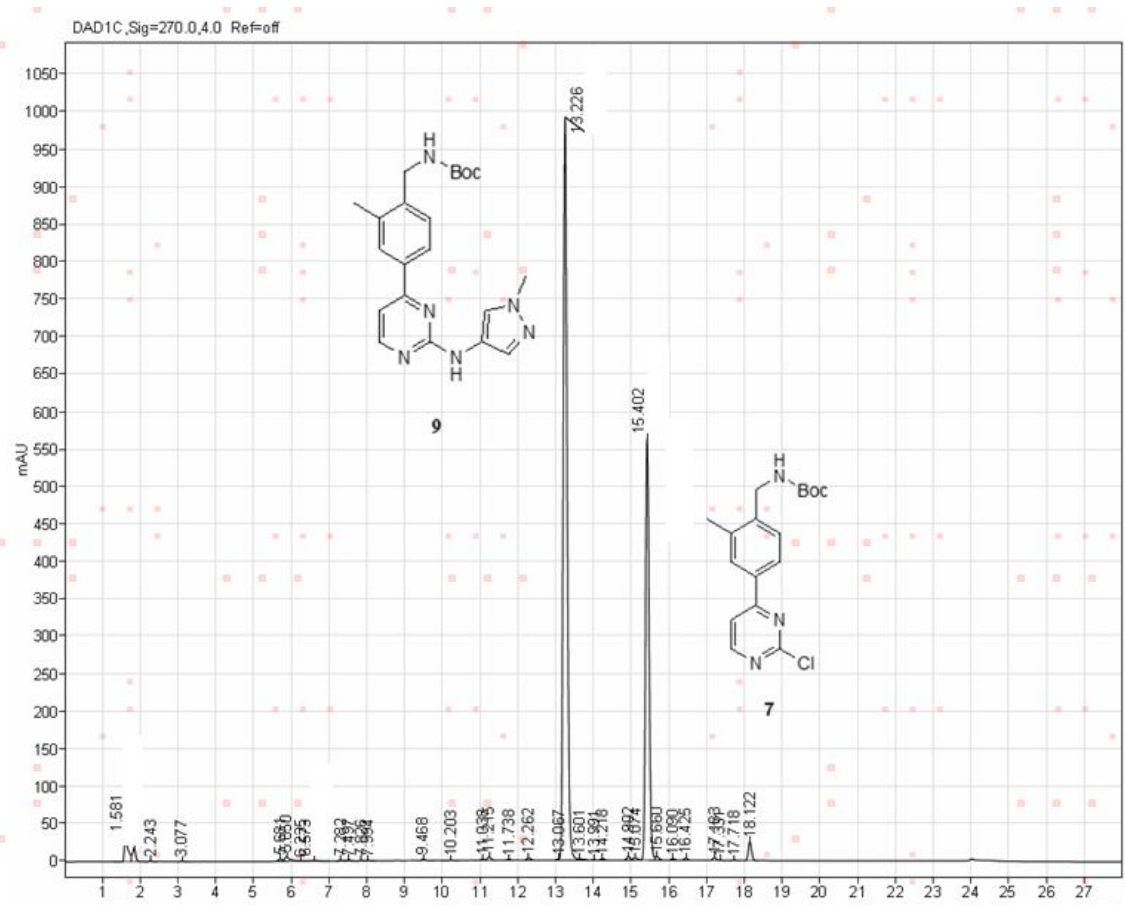

$\begin{array}{ccccc}\text { RT [min] } & \text { Area } & \text { Area\% } & \text { Resolution USP } & \text { Height(mAU } \\ 2.243 & 6.87 & 0.06 & 2.8 & 1.3 \\ 3.077 & 4.48 & 0.04 & 5.5 & 0.7 \\ 5.681 & 21.93 & 0.19 & 13.9 & 3.2 \\ 5.850 & 57.76 & 0.49 & 0.8 & 5.9 \\ 6.235 & 4.88 & 0.04 & 1.6 & 0.5 \\ 6.573 & 8.38 & 0.07 & 1.2 & 0.7 \\ 7.282 & 10.80 & 0.09 & 2.4 & 1.2 \\ 7.497 & 12.93 & 0.11 & 0.9 & 1.7 \\ 7.826 & 3.49 & 0.03 & 1.8 & 0.6 \\ 7.994 & 5.01 & 0.04 & 0.7 & 0.5 \\ 9.468 & 15.23 & 0.13 & 6.4 & 2.2 \\ 10.203 & 5.10 & 0.04 & 3.9 & 0.6 \\ 11.038 & 12.35 & 0.11 & 4.3 & 1.6 \\ 11.215 & 33.81 & 0.29 & 0.9 & 4.8 \\ 11.738 & 10.17 & 0.09 & 2.5 & 1.2 \\ 12.262 & 25.42 & 0.22 & 2.5 & 3.2 \\ 13.067 & 8.50 & 0.07 & & 1.9 \\ 13.226 & 7027.41 & 60.07 & & 991.5 \\ 13.601 & 22.48 & 0.19 & 0.6 & 2.1 \\ 13.991 & 5.30 & 0.05 & 0.6 & 0.6 \\ 14.218 & 16.33 & 0.14 & 1.0 & 2.2 \\ 14.902 & 30.29 & 0.26 & 3.8 & 4.8 \\ 15.074 & 26.53 & 0.23 & 0.8 & 2.9 \\ 15.402 & 3729.13 & 31.88 & 1.5 & 562.7\end{array}$


Entry 8 HPLC spectra

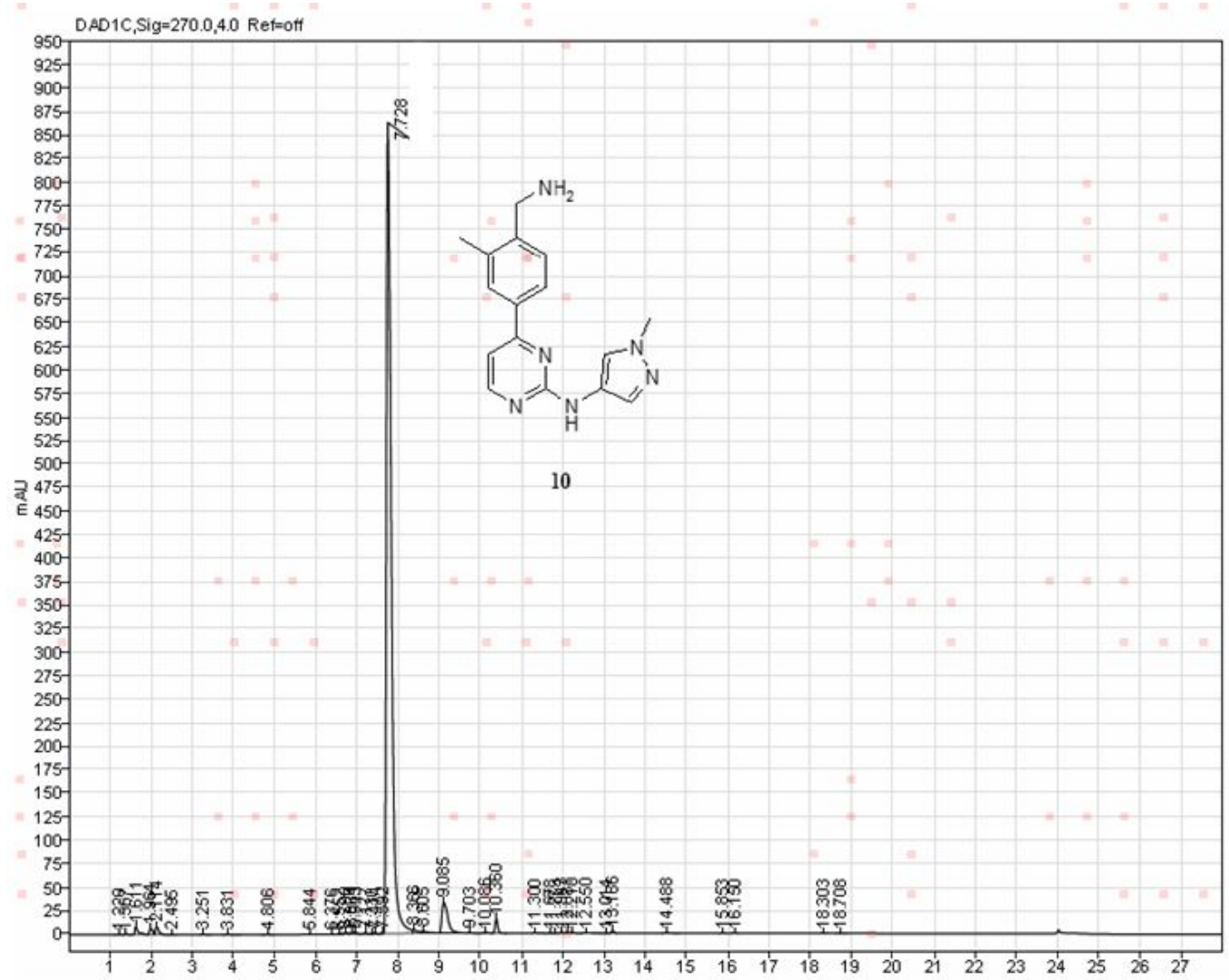

$\begin{array}{lcccc}\text { RT [min] } & \text { Area } & \text { Area\% } & \text { Resolution USP } & \text { Height(mAU) } \\ 1.964 & 37.45 & 0.48 & 7.0 \\ 2.114 & 50.17 & 0.64 & 8.9 \\ 2.495 & 1.43 & 0.02 & 0.2 \\ 3.251 & 3.67 & 0.05 & 0.7 \\ 3.831 & 4.90 & 0.06 & 0.8 \\ 4.806 & 2.89 & 0.04 & 0.4 \\ 5.844 & 2.78 & 0.04 & 0.3 \\ 6.376 & 1.09 & 0.01 & 0.2 \\ 6.552 & 3.53 & 0.05 & 0.6 \\ 6.700 & 11.41 & 0.15 & 1.6 \\ 6.864 & 10.22 & 0.13 & 2.3 \\ 6.943 & 8.93 & 0.11 & 2.2 \\ 7.177 & 4.47 & 0.06 & 1.1 \\ 7.330 & 0.71 & 0.01 & 0.3 \\ 7.414 & 6.31 & 0.08 & 1.6 \\ 7.592 & 3.94 & 0.05 & 1.2 \\ 7.728 & 7120.78 & 90.76 & 862.8\end{array}$


Entry 9 HPLC spectra

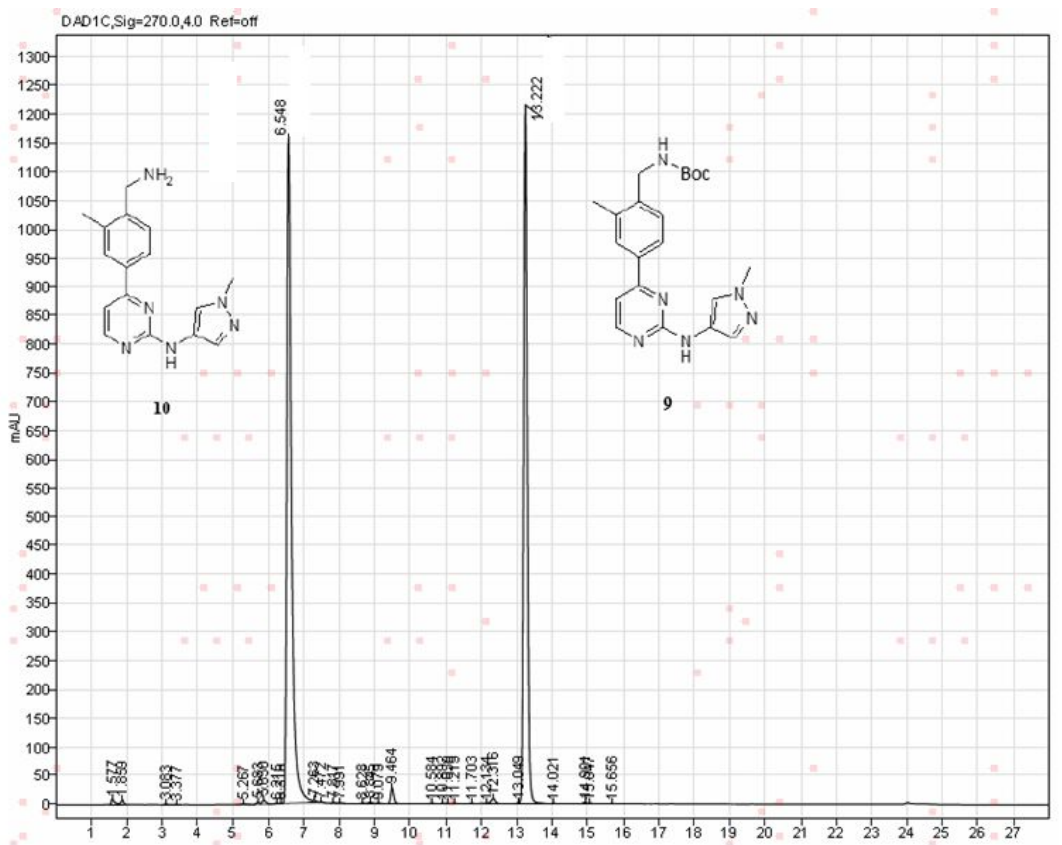

\begin{tabular}{|c|c|c|c|c|c|}
\hline RT [min] & & Area & Area $\%$ & Resolution USP & Height( mAU) \\
\hline 3083 & $=$ & 8.41 & 0.04 & 7.8 & 1.2 \\
\hline 3.377 & & 5.64 & 0.03 & 1.6 & 0.7 \\
\hline 5267 & & 15.58 & 0.08 & 7.9 & 1.4 \\
\hline 5683 & & 23.56 & 0.12 & 1.7 & 3.5 \\
\hline 5850 & & 55.48 & 0.28 & 0.8 & 7.3 \\
\hline 6215 & & 8.26 & 0.04 & 2.0 & 1.5 \\
\hline 6.318 & & 3.68 & 0.02 & 0.3 & 0.8 \\
\hline 6.548 & $=$ & 10598.03 & 53.78 & 0.6 & 1155.6 \\
\hline 7263 & $=$ & 25.72 & 0.13 & 2.2 & 3.7 \\
\hline 7.472 & & 4.78 & 0.02 & 0.7 & 0.9 \\
\hline 7817 & & 7.00 & 0.04 & 2.5 & 1.3 \\
\hline 7991 & & 7.22 & 0.04 & 1.0 & 0.9 \\
\hline 8.628 & & 9.69 & 0.05 & 1.7 & 1.0 \\
\hline 8845 & & 2009 & 0.10 & 0.5 & 2.1 \\
\hline 9079 & & 10.11 & 0.05 & 1.1 & 1.4 \\
\hline 9.464 & & 166.58 & 0.85 & 2.3 & 28.0 \\
\hline 10.584 & & 9.68 & 0.05 & 6.0 & 1.0 \\
\hline 10.892 & & 3.92 & 0.02 & 1.5 & 0.6 \\
\hline 11.030 & $=$ & 3.78 & 0.02 & 0.7 & 0.6 \\
\hline 11.219 & & 7.27 & 0.04 & 1.0 & 1.1 \\
\hline 11.703 & & 4.84 & 0.02 & 2.3 & 0.8 \\
\hline 12.134 & & 6.35 & 0.03 & 1.2 & 1.0 \\
\hline 12.316 & & 73.78 & 0.37 & 0.5 & 9.1 \\
\hline 13.049 & & 6.98 & 0.04 & & 2.2 \\
\hline 13.222 & & 8467.17 & 42.97 & & 1215.5 \\
\hline
\end{tabular}


Entry 10 HPLC spectra

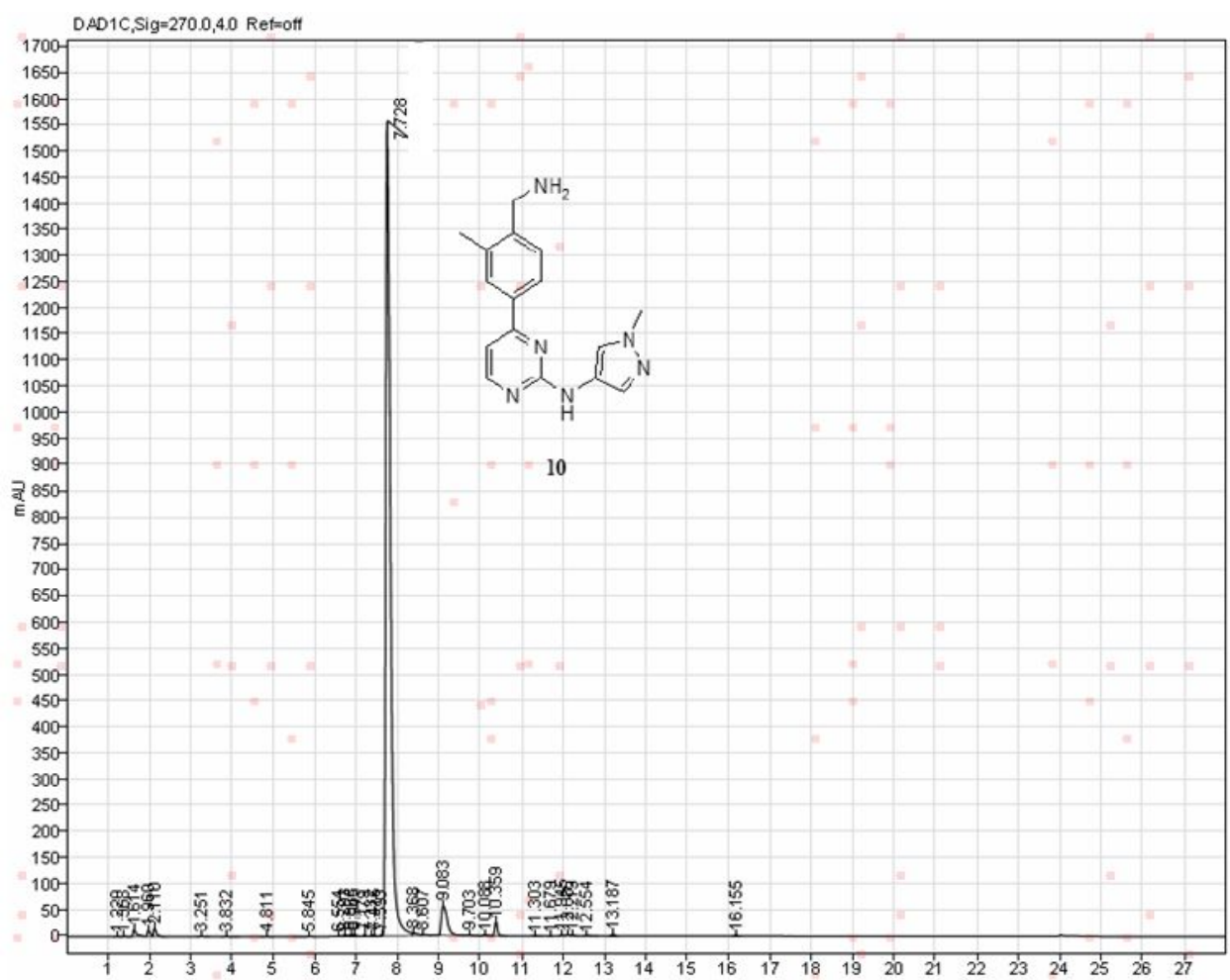

$\begin{array}{ccccc}\text { RT [min] } & \text { Area } & \text { Area\% } & \text { Resolution USP } & \text { Height(mAU) } \\ 1.960 & 57.47 & 0.42 & 2.7 & 12.2 \\ 2.110 & 78.21 & 0.57 & 1.2 & 16.1 \\ 3.251 & 6.49 & 0.05 & 9.0 & 1.3 \\ 3.832 & 8.39 & 0.06 & 4.1 & 1.4 \\ 4.811 & 5.01 & 0.04 & 6.1 & 0.7 \\ 5.845 & 6.71 & 0.05 & 4.6 & 0.6 \\ 6.554 & 6.32 & 0.05 & 2.8 & 1.1 \\ 6.703 & 20.13 & 0.15 & 0.7 & 2.9 \\ 6.865 & 17.90 & 0.13 & 0.9 & 4.0 \\ 6.945 & 16.94 & 0.12 & 0.6 & 4.2 \\ 7.179 & 7.94 & 0.06 & 2.0 & 2.0 \\ 7.332 & 1.29 & 0.01 & 1.3 & 0.5 \\ 7.415 & 11.04 & 0.08 & 0.7 & 2.7 \\ 7.593 & 6.88 & 0.05 & 1.7 & 2.2 \\ 7.728 & 12462.65 & 91.15 & 0.9 & 1558.0\end{array}$


Entry 11 HPLC spectra

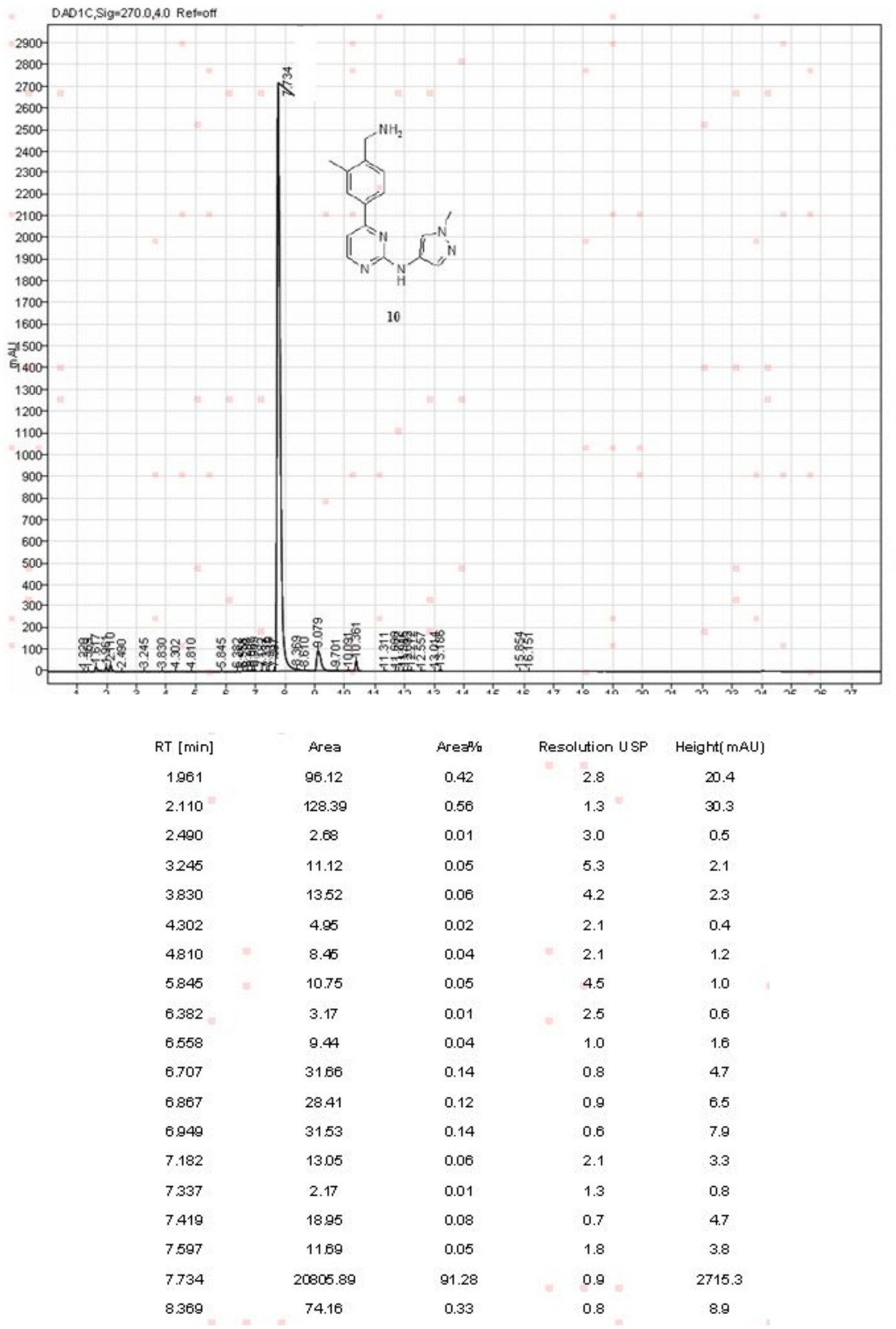


Entry 12 HPLC spectra

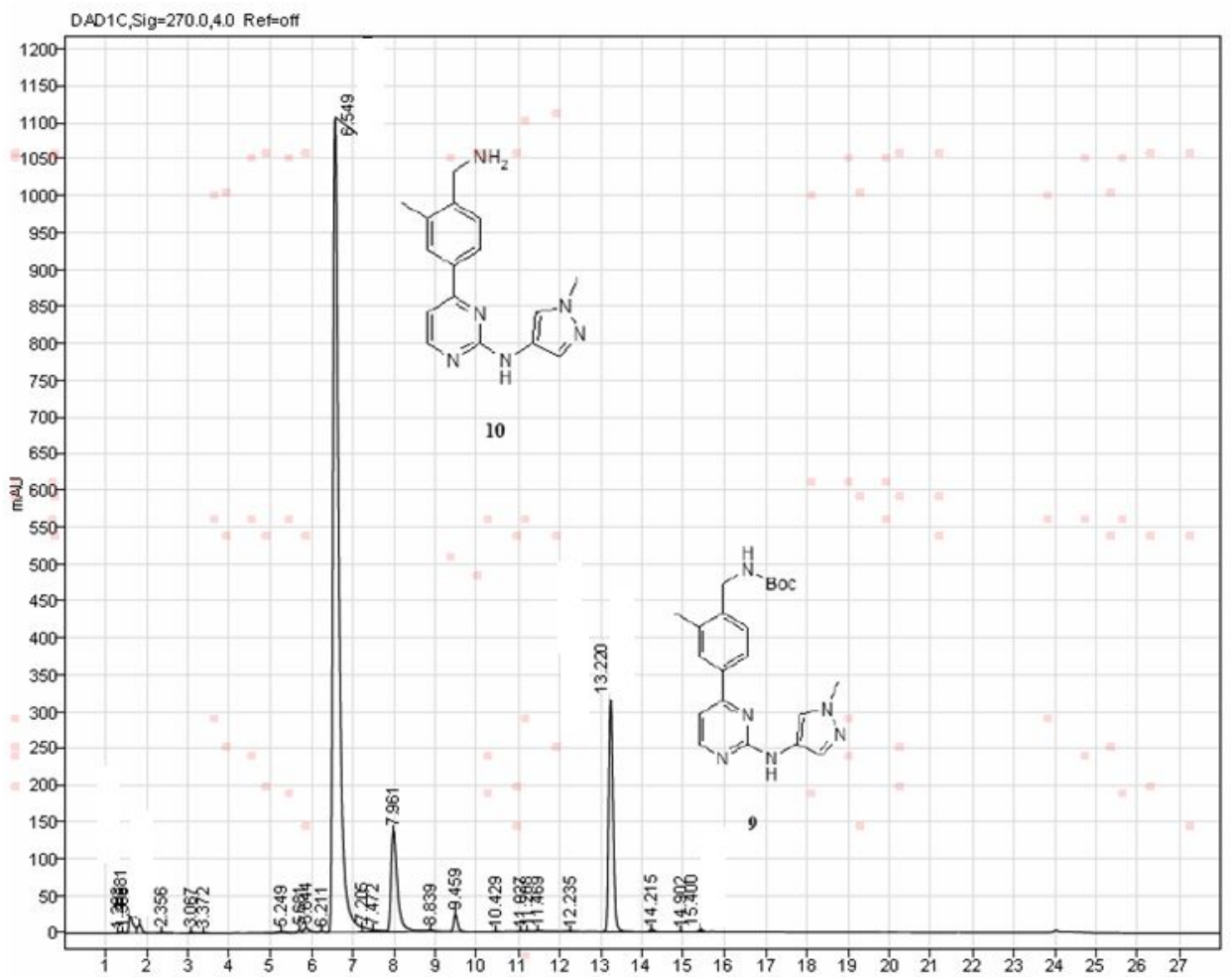

$\begin{array}{lcccc}\text { RT [min] } & \text { Area } & \text { Area\% } & \text { Resolution USP } & \text { Height(mAU] } \\ 1.581 & 173.44 & 1.11 & 1.3 & 25.3 \\ 1811 & 81.95 & 0.52 & 1.2 & 11.6 \\ 2.356 & 6.11 & 0.04 & 3.0 & 1.0 \\ 3.067 & 8.73 & 0.06 & 4.2 & 1.2 \\ 3.372 & 5.65 & 0.04 & 1.5 & 0.6 \\ 5.249 & 30.96 & 0.20 & 6.5 & 2.2 \\ 5.681 & 17.37 & 0.11 & 1.5 & 2.5 \\ 5844 & 5685 & 0.36 & 0.8 & 6.2 \\ 6211 & 10.10 & 0.06 & 1.9 & 1.6 \\ 6.549 & 11104.57 & 70.77 & 1.6 & 1106.7 \\ 7205 & 68.09 & 0.43 & & 6.3 \\ 7.472 & 36.53 & 0.23 & & 2.9 \\ 7.961 & 1477.75 & 9.42 & & 136.7 \\ 8.839 & 29.39 & 0.19 & 3.0 & 2.1 \\ 9.459 & 150.10 & 0.96 & 2.5 & 23.5 \\ 10.429 & 4.93 & 0.03 & 5.0 & 0.6 \\ 11.027 & 5.80 & 0.04 & 2.8 & 0.8 \\ 11.208 & 5.98 & 0.04 & 1.0 & 0.9 \\ 11.469 & 1483 & 0.09 & 1.1 & 1.3 \\ 12.236 & 8.97 & 0.06 & 2.5 & 0.7 \\ 13.220 & 2339.34 & 14.91 & 3.7 & 316.9\end{array}$


Entry 13 The HPLC spectra

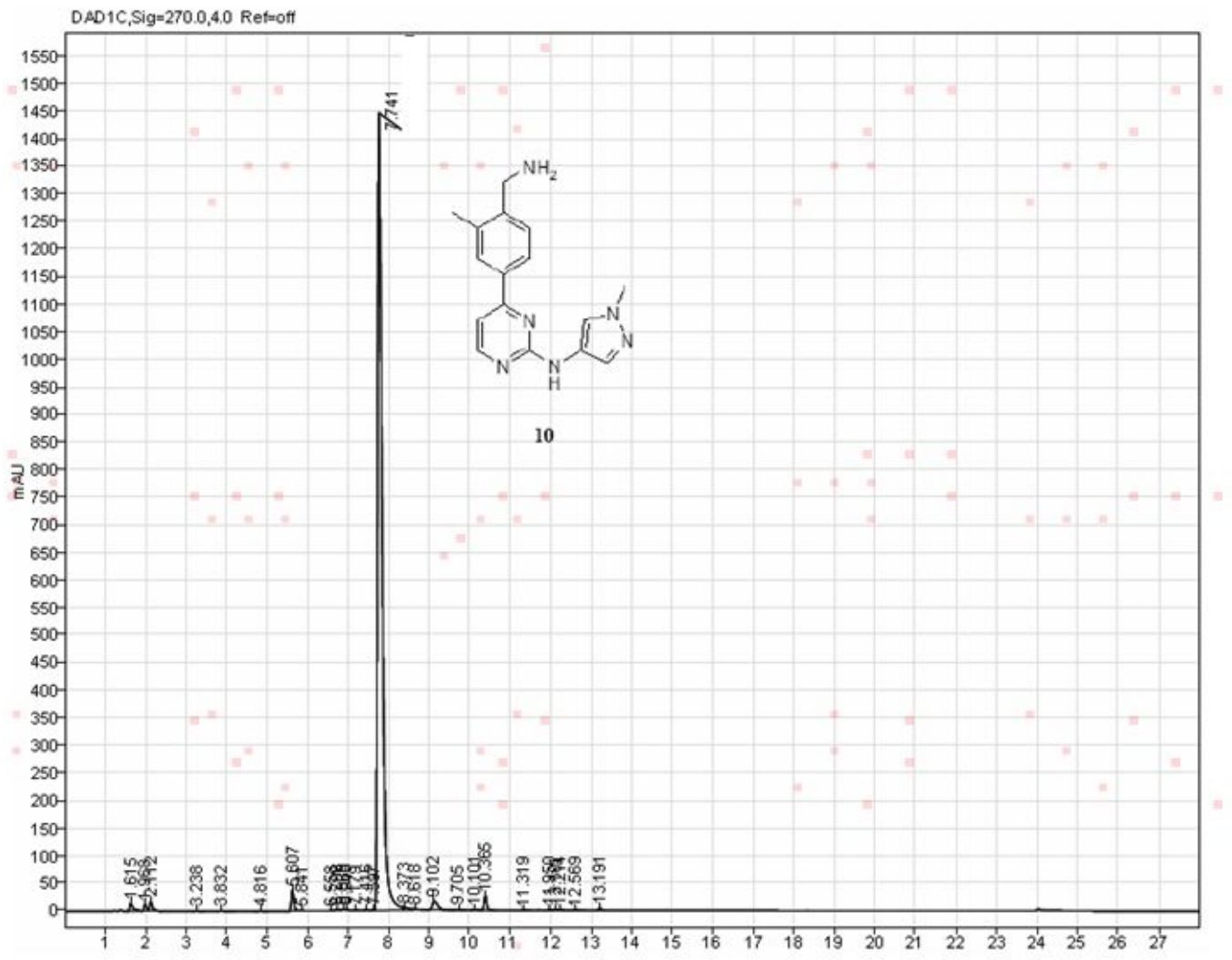

$\begin{array}{ccccc}\text { RT [min] } & \text { Area } & \text { Area\% } & \text { Resolution USP } & \text { Height(mAU) } \\ 1.738 & 100.01 & 0.87 & 21.1 \\ 2.052 & 1.91 & 0.02 & 0.4 \\ 2.165 & 2.24 & 0.02 & 0.3 \\ 2.590 & 3.18 & 0.03 & 0.3 \\ 2.914 & 11.30 & 0.10 & 1.3 \\ 3.110 & 3.64 & 0.03 & 0.5 \\ 4.210 & 3.12 & 0.03 & 0.3 \\ 4.754 & 22.29 & 0.19 & 1.5 \\ 5.412 & 58.82 & 0.51 & 5.4 \\ 5.812 & 7.70 & 0.07 & 1.1 \\ 6.176 & 10898.34 & 95.04 & 1118.1 \\ 7.646 & 36.55 & 0.32 & 2.2 \\ 8.462 & 36.99 & 0.32 & 2.5 \\ 9.078 & 114.78 & 1.00 & 20.6\end{array}$


Entry 14 HPLC spectra

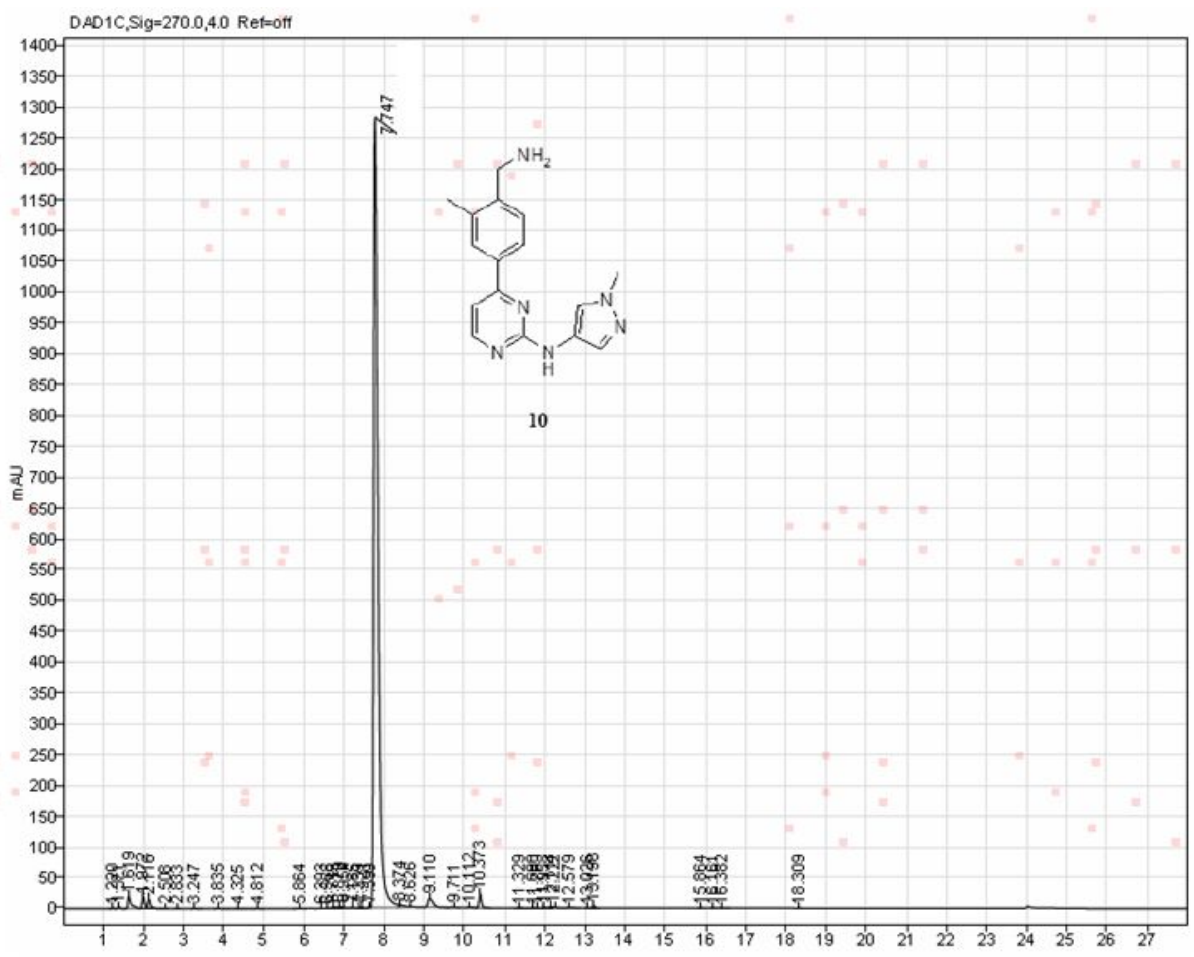

$\begin{array}{lcccc}\text { RT [min] } & \text { Area } & \text { Area\% } & \text { Resolution USP } & \text { Height(mAU) } \\ 1.972 & 86.73 & 0.81 & 2.9 & 19.3 \\ 2.116 & 75.72 & 0.71 & 1.2 & 15.8 \\ 2.508 & 1.93 & 0.02 & 2.7 & 0.3 \\ 2.833 & 0.59 & 0.01 & 2.3 & 0.2 \\ 3.247 & 5.26 & 0.05 & 3.6 & 1.1 \\ 3.835 & 7.09 & 0.07 & 4.2 & 1.1 \\ 4.325 & 2.58 & 0.02 & 2.4 & 0.3 \\ 4.812 & 3.87 & 0.04 & 2.3 & 0.6 \\ 5.864 & 3.74 & 0.03 & 5.2 & 0.4 \\ 6.393 & 1.74 & 0.02 & 2.7 & 0.3 \\ 6.568 & 4.94 & 0.05 & 1.0 & 0.9 \\ 6.719 & 14.00 & 0.13 & 0.7 & 2.1 \\ 6.870 & 14.55 & 0.14 & 0.9 & 3.2 \\ 6.954 & 10.36 & 0.10 & 0.7 & 2.5 \\ 7.185 & 4.36 & 0.04 & 2.0 & 1.1 \\ 7.339 & 1.12 & 0.01 & 1.4 & 0.4 \\ 7.421 & 8.25 & 0.08 & 0.7 & 2.1 \\ 7.599 & 3.75 & 0.03 & 2.0 & 1.3 \\ 7.747 & 9949.19 & 92.84 & 1.0 & 1283.0\end{array}$


Entry 15 HPLC spectra

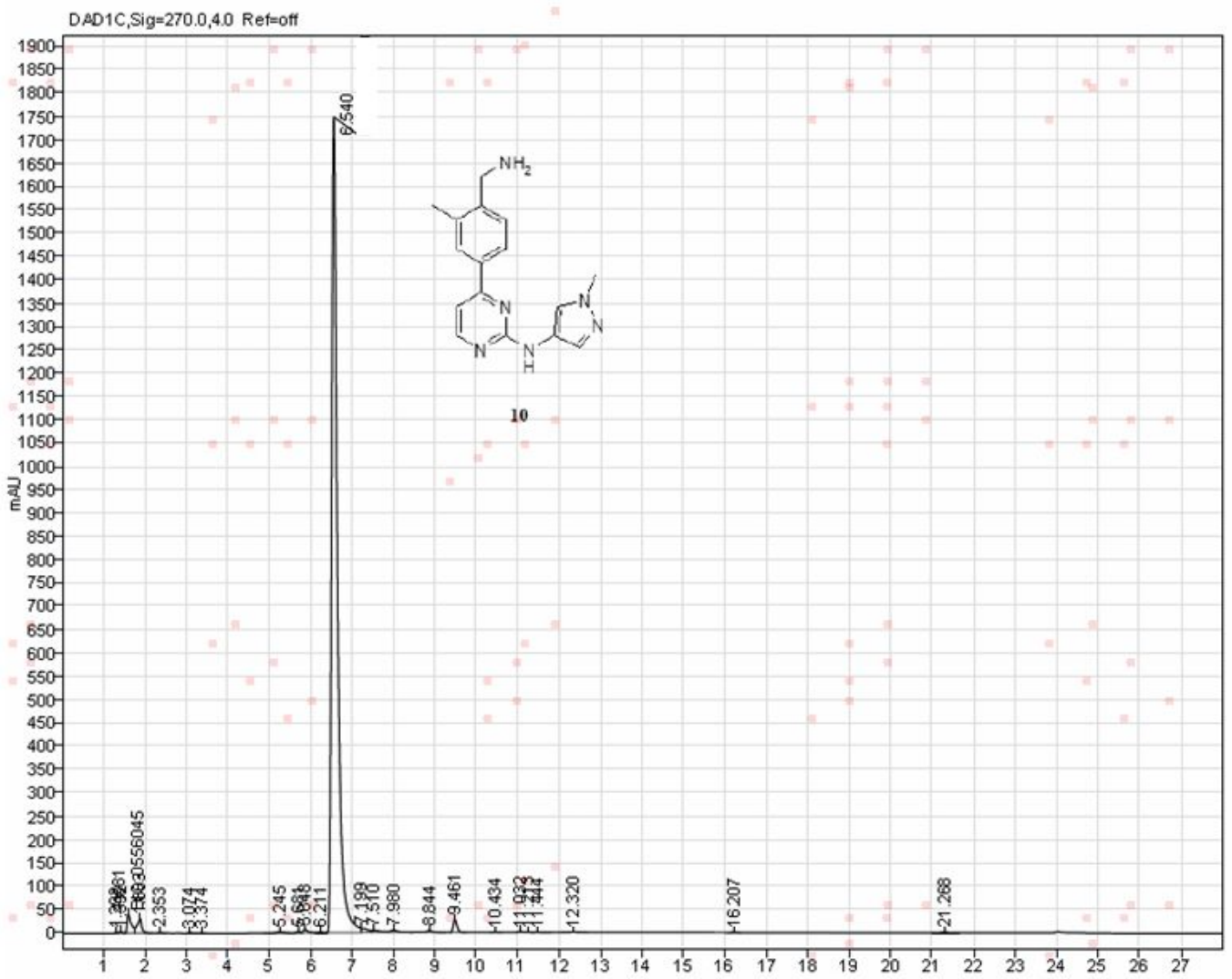

$R T$ [m in
1.581
1.863
2.353
3.074
3.374
5.245
5.681
5.848
6.211
6.540

Area
273.67
241.39
8.28
11.11
5.65
37.27
20.51
60.00
10.65
17082.10

A rea\%

1.50

1.32

0.05

0.06

0.03

0.20

0.11

0.33

0.06

93.43

\section{Resolution USP Height(mAU)}

$\begin{array}{lr}1.3 & 40.8 \\ 1.7 & 31.3 \\ 3.0 & 1.5 \\ 4.2 & 1.4 \\ 1.5 & 0.7 \\ 6.8 & 2.6 \\ 1.6 & 3.1 \\ 0.8 & 7.1 \\ 2.0 & 1.7 \\ 1.6 & 1748.9\end{array}$




\section{Single Crystal Structure of 19}

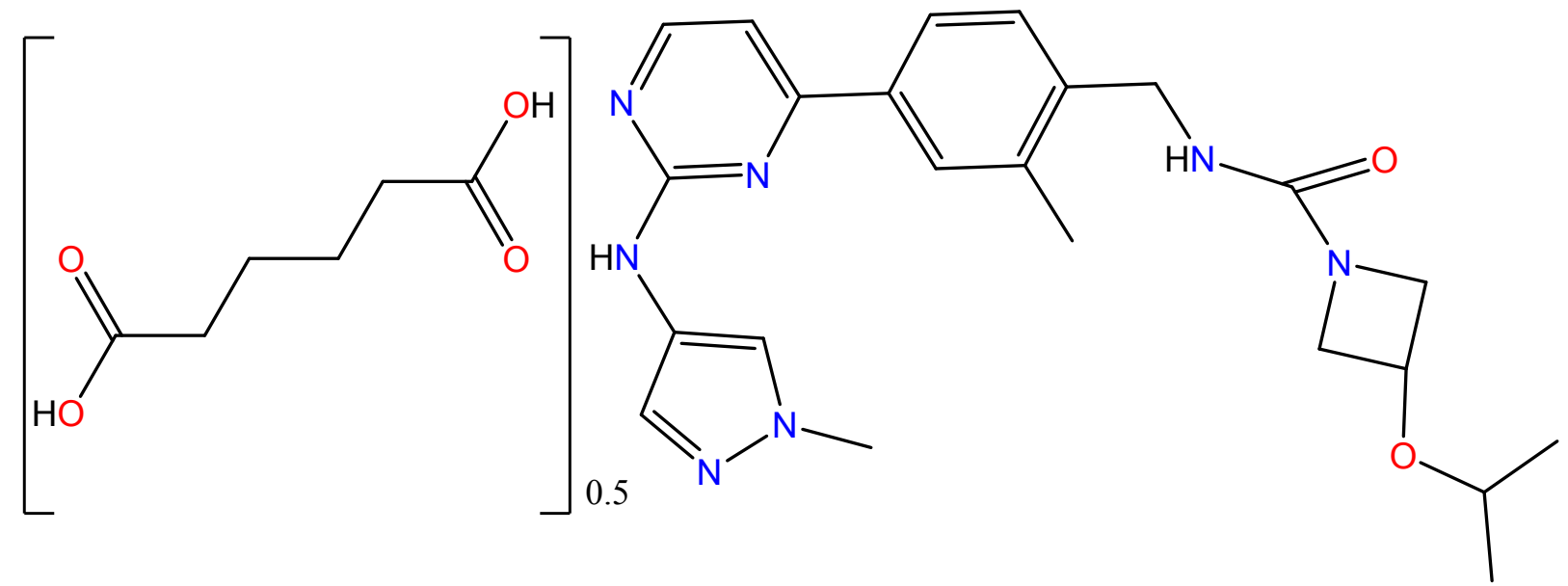

Figure S1: Chemical structure of BIIB068 hemi-adipic acid cocrystal
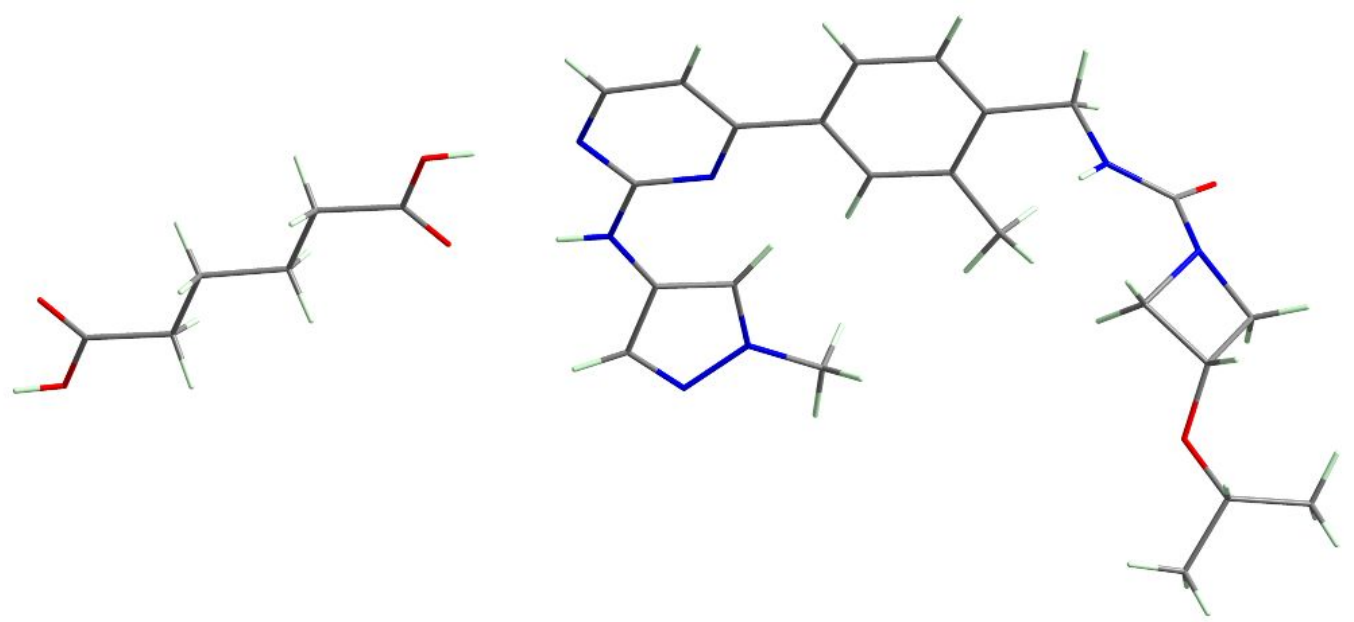

Figure S2: Three-dimensional structure of BIIB068 hemi-adipic acid cocrystal single crystal (I) 

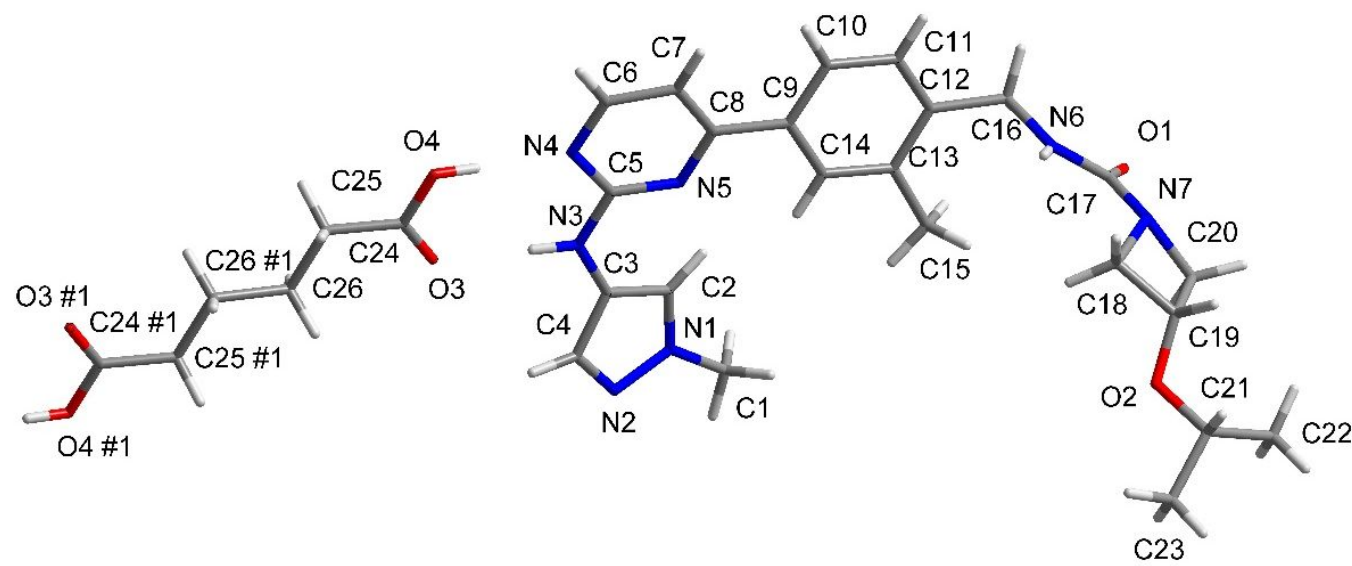

Figure S3: Three-dimensional structure of BIIB068 hemi-adipic acid cocrystal single crystal (II)
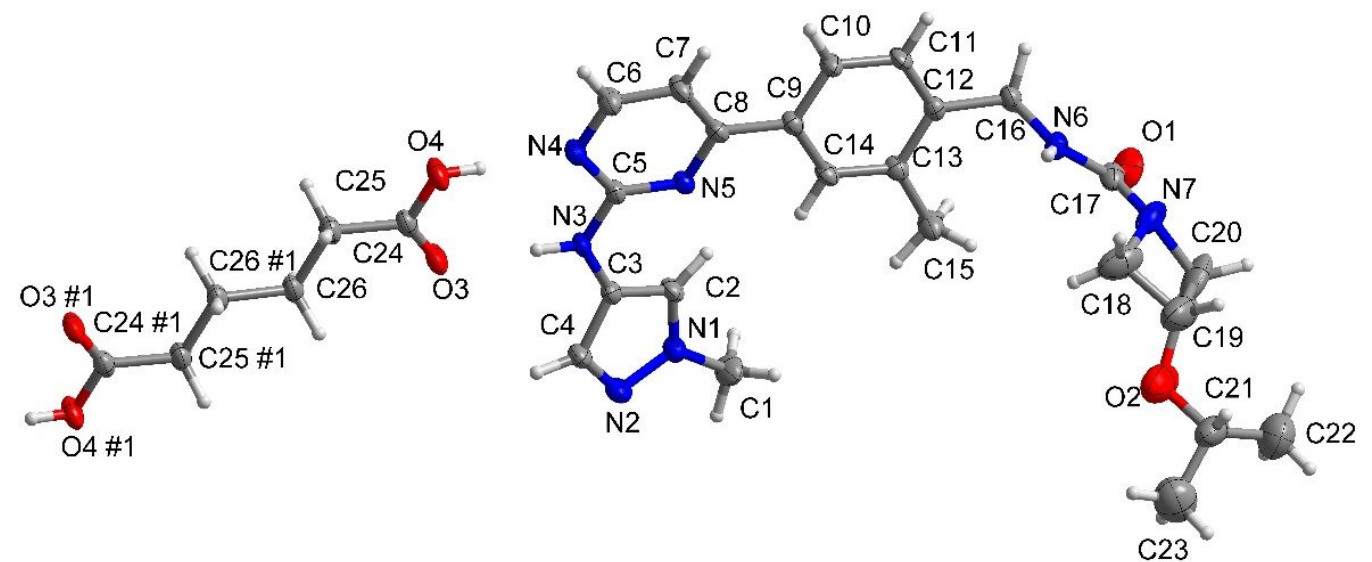

Figure S1: The ORTEP structure (50\% probability) of BIIB068 hemi-adipic acid cocrystal single crystal 


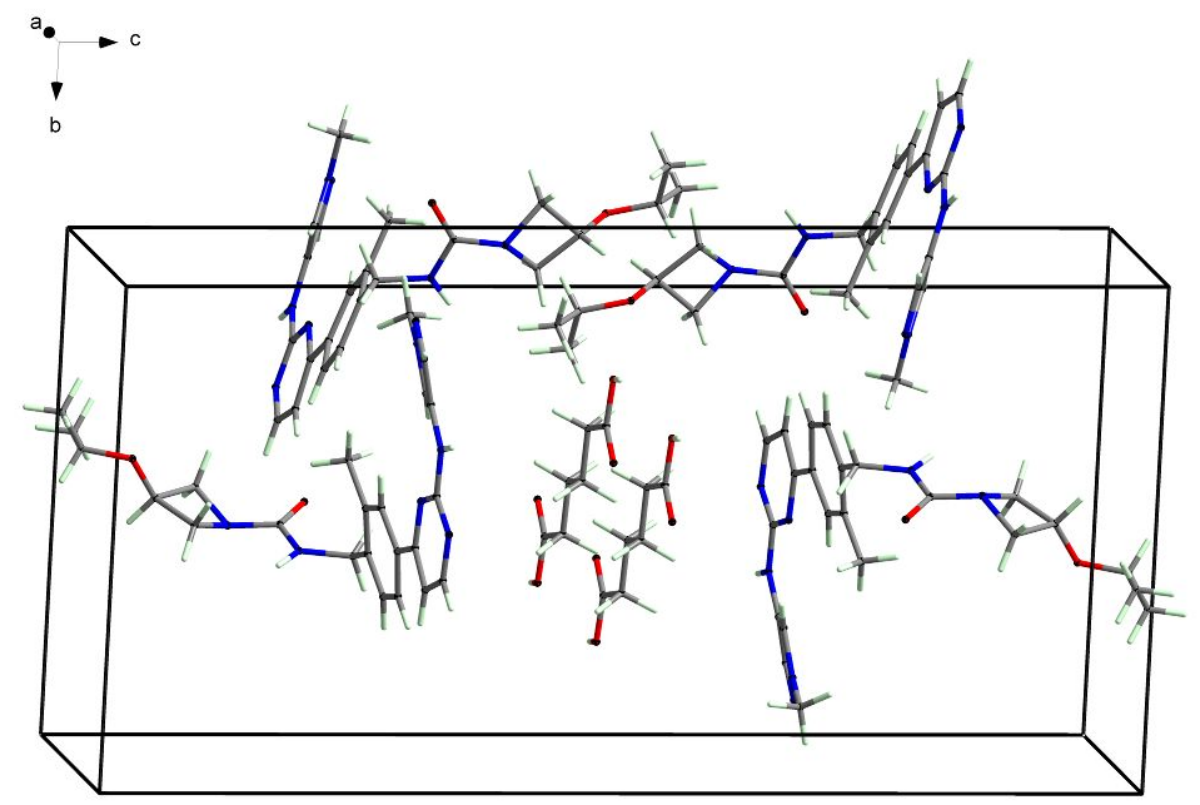

Figure S5: Unit cell of BIIB068 hemi-adipic acid cocrystal single crystal

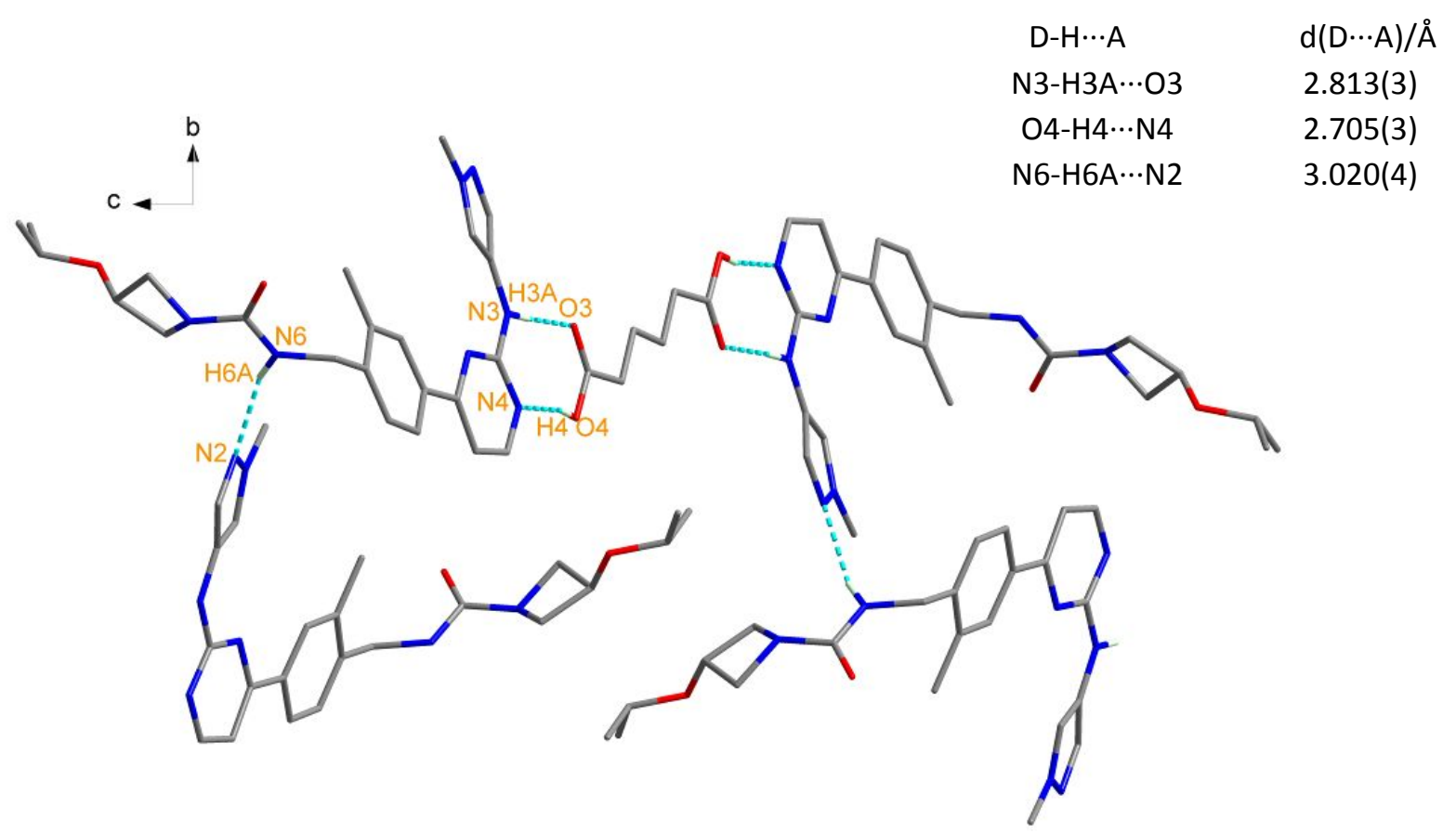

Figure S6: Hydrogen bonds of BIIB068 hemi-adipic acid cocrystal single crystal (Other hydrogen atoms were omitted for clarity) 


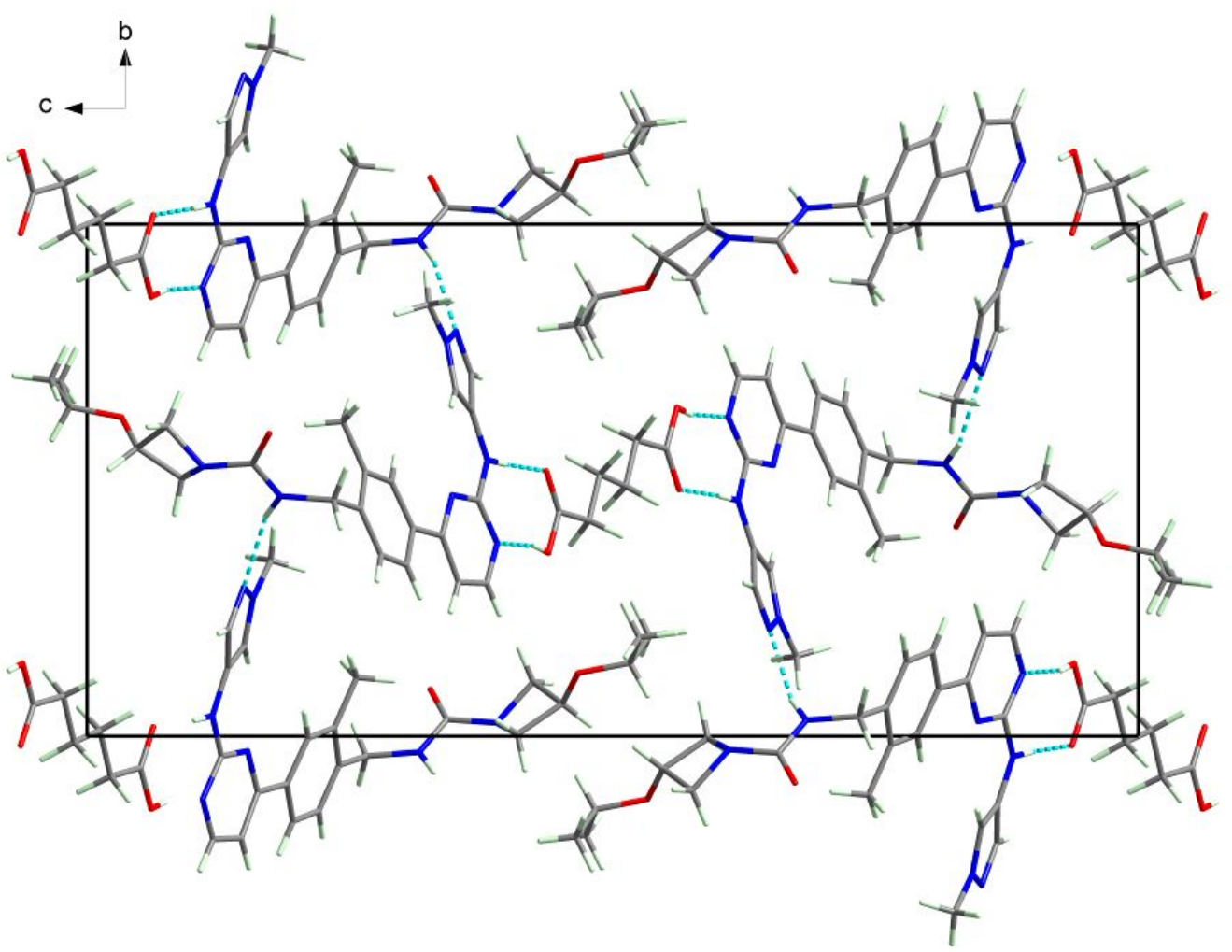

Figure S7: Crystal packing of BIIB068 hemi-adipic acid cocrystal single crystal view along $a$-axis

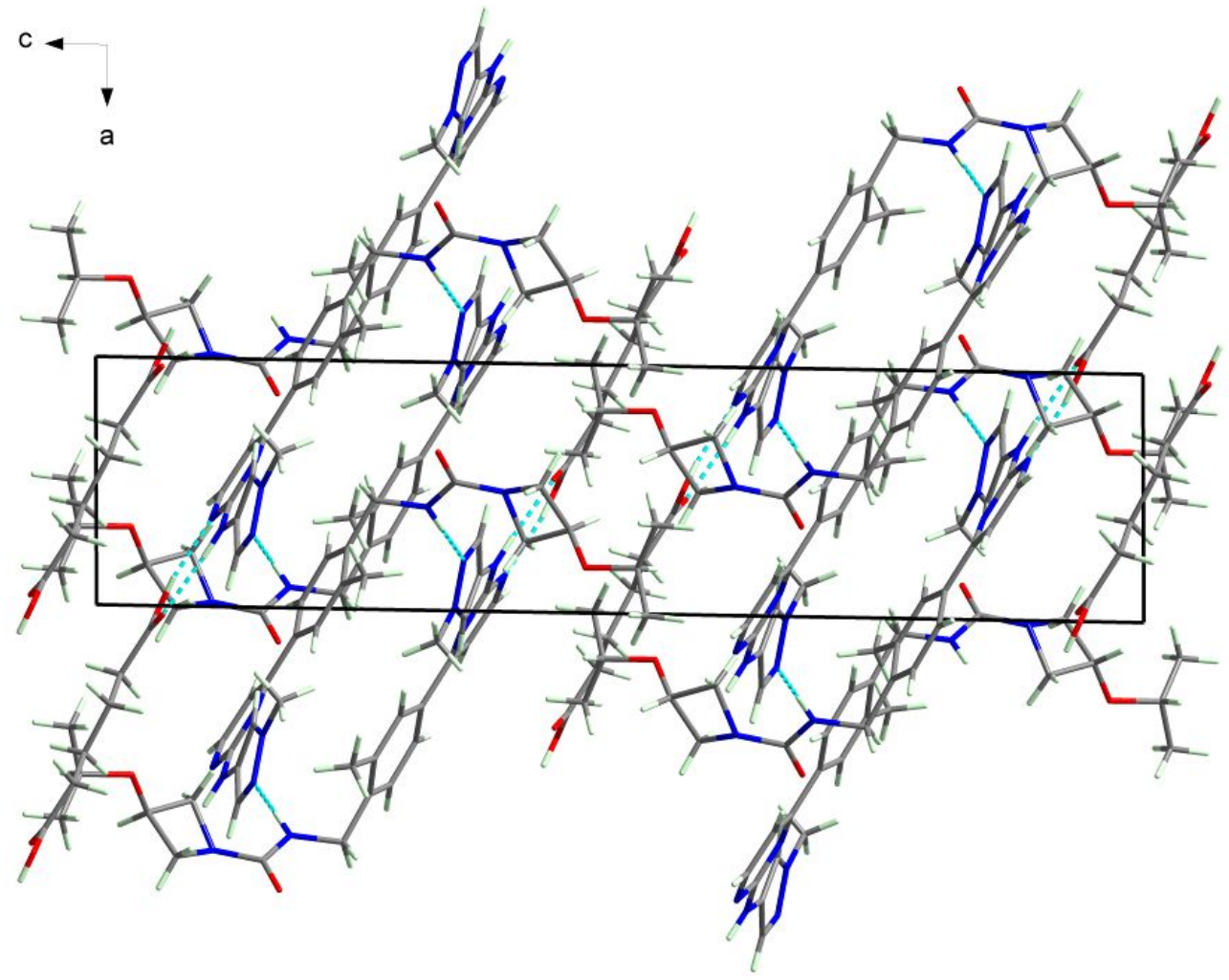

Figure S2: Crystal packing of BIIB068 hemi-adipic acid cocrystal single crystal view along $b$-axis 


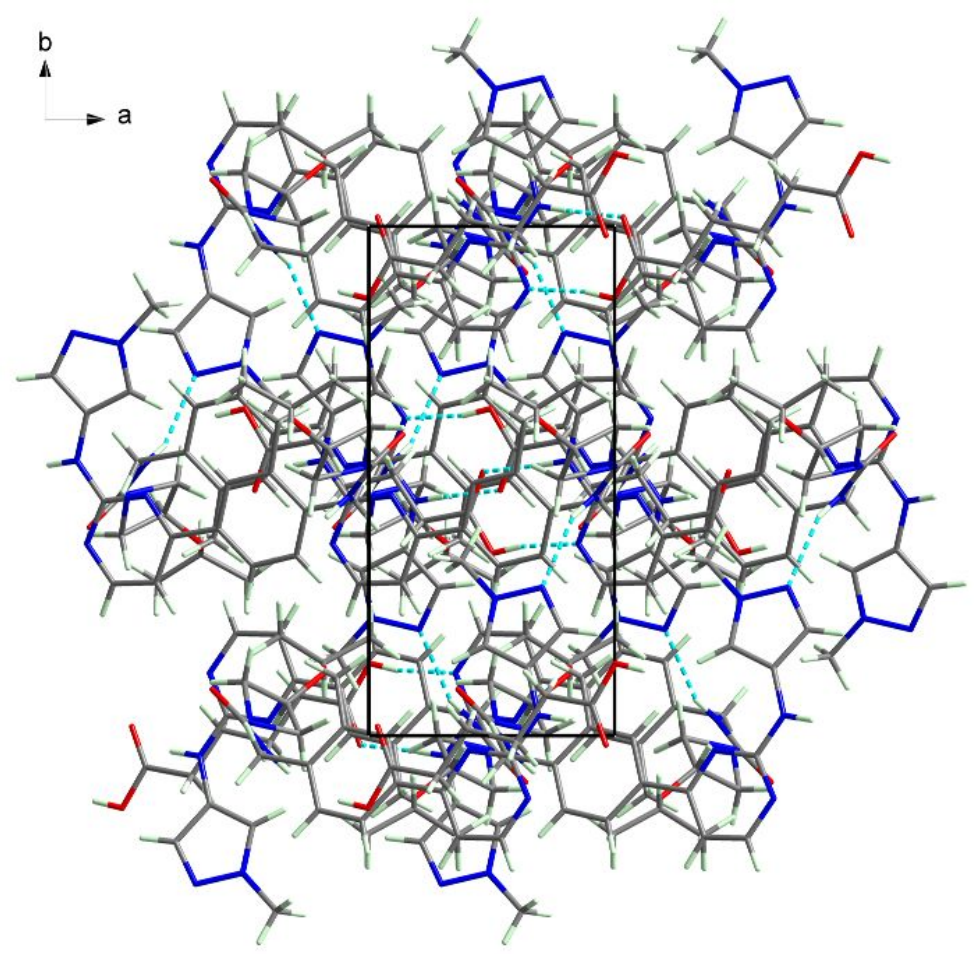

Figure S3: Crystal packing of BIIB068 hemi-adipic acid cocrystal single crystal view along $c$-axis 